\title{
On the Tamagawa number conjecture for Hecke characters
}

\author{
Francesc Bars *
}

October 16, 2018

\begin{abstract}
In this paper we prove the weak $p$-part of the Tamagawa number conjecture in all non-critical cases for the motives associated to Hecke characters of the form $\varphi^{a} \bar{\varphi}^{b}$ where $\varphi$ is the Hecke character of a CM elliptic curve $E$ defined over an imaginary quadratic field $K$, under certain restrictions which originate mainly from the Iwasawa theory of imaginary quadratic fields.
\end{abstract}

\section{Introduction}

The Tamagawa number conjecture for a variety X over a number field of Bloch and Kato [5], or, more precisely, for a motive $M$ of pure weight $w$ over a number field, describes the special values of the $L$-function in terms of cohomological data (see for example Kato [19] or Fontaine and Perrin-Riou [11]) and the ppart of the conjecture describes these values up to units in the ring $\mathbb{Z}_{(p)}:=\left\{\frac{a}{b} \in\right.$ $\mathbb{Q} \mid a, b \in \mathbb{Z}, b \neq 0,(b, p)=1\}$.

Recall that the special values of an $L$-function are the leading coefficient of Taylor expansion at integer points. Suppose we have a motive $M$ of weight $w$ such that it's $L$-function has meromorphic continuation and satisfies the expected functional equation. We say that an integer $m<\frac{w}{2}$ is non-critical if $L(M, m)=0$ and that it is critical if $L(M, m) \neq 0$. We extend this definition to the integers $m>\frac{w}{2}+1$ by saying that $m$ is critical for $M$ if $w-m+1$ is critical for $\check{M}$ the dual motive associated to $M$, and non-critical for $M$ if $w-m+1$ is non-critical for $\check{M}$. The Tamagawa number conjecture can be formulated in terms of period maps (period integrals nor $p$-adic periods appears) and regulator maps $([10,19])$, but in the non-critical situation it can be formulated for almost all non-critical cases (using the hypothetical functional equation and good compatibilities) without the period maps, where by period map we mean a map between de Rham to Betti cohomology and for the $p$-adic period a map between étale cohomology to de Rham cohomology (see [19, §2.3]).

There are few cases proved in the non-critical situation: for the Riemann zeta function $([5, \S 6])$, for Dirichlet motives ([6], [17]), for CM elliptic curves defined over the field of the endomorphism ring ([22]) or defined over $\mathbb{Q}([5, \S 7]$, [1]).

*Work partially supported by BFM2003-06092 
The weak $p$-part of the Tamagawa number conjecture for an elliptic curve $E$ with CM by $K$ defined over the field of endomorphisms, proved by Kings [22], is related to the weak $p$-part of the Tamagawa number conjecture for the $L$ function of the Hecke character $\varphi$, associated to $E$, over the imaginary quadratic field $K$ of class number 1 . More precisely, Kings proves in 22 the conjecture for the motive $h(\bar{\varphi})(-r)$ with $r \geq 0$ which corresponds to the special value (non-critical) for the $L$ function associated to $\bar{\varphi}$ at $-r$, where $h(\bar{\varphi})$ is the motive associated to $\bar{\varphi}$ over $K$ with $K$-coefficients. As a consequence, he obtains the conjecture for the motive $h^{1}(E)(-r)$. Using the functional equation for $E$ and good compatibilities one should obtain the conjecture for $h^{1}(E)(r+2)$. We generalize the methods of Kings to other Hecke characters over an imaginary quadratic field $K$ in the non-critical situation.

We consider the motive associated to the Hecke character $\varphi^{a} \bar{\varphi}^{b}$ with $a, b \geq 0$, which has weight $a+b$. It is known that almost all the non-critical values for this motive are the integers lower than $\min (a, b)$. Our work is concentrated in this situation, but we remark that there are results on the Tamagawa number conjecture in the critical situation (Harrison [16], Guo [14, Kimura [23], Han [15] and in greater generality by Tsuji 27]).

The aim of this paper is to prove the $p$-part of the Tamagawa number conjecture (under the formulation in [19, §2.2]) for all the non-critical values for the $L$-function of the motive associated Hecke characters $\psi_{\theta}$ of an imaginary quadratic field $K$ with class number 1, which under a fixed embedding corresponds to $\varphi^{a} \bar{\varphi}^{b}$.

The main results of this paper are Theorems 5.125 .13 and Theorems 6.3 , 6.4 These results are the weak $p$-part of the Tamagawa number conjecture for the geometric object associated to the Hecke characters over $K$ with $a \not \equiv$ $b\left(\operatorname{modulo}\left(\# \mathcal{O}_{K}^{*}\right)\right)$ with $K$ or $\mathbb{Q}$-coefficients, under certain restrictions which originate mainly from Iwasawa theory of imaginary quadratic fields, where $\mathcal{O}_{K}^{*}$ means the invertible elements of the ring of integers of $K$. See the last section for numerical examples.

To obtain these main results, we study in detail the image with respect to the regulator map of a certain non-trivial submodule of some $K$-theory group. The basic ingredients used in the proof of these results are Deninger's proof of the Beilinson's conjecture for Hecke characters in [8], the specialization of the polylogarithm sheaf [22] and the Iwasawa main conjecture for imaginary quadratic fields 24], as in [22]. This paper need to deal with negative twists. This problem does not appear in [22. For negative twists, we modify Deninger's elements [8] in order to apply the $p$-adic techniques of [22].

The results of this paper generalize the results in [2, Chapter 3] which restricts to the Hecke characters $\bar{\varphi}^{m}$.

\section{The motive associated to Hecke characters}

Let $K$ be an imaginary quadratic field with class number $\operatorname{cl}(K)$ equal to 1 and $\mathcal{O}_{K}$ be its ring of integers. Let $D_{K}$ be the discriminant of $K$. Let $E$ be an elliptic curve over $K$ with $\mathrm{CM}$ by $\mathcal{O}_{K}$. In this section we describe some pure motives coming from a self product of the motive $h^{1}(E)$ and their realizations, and we prove that the $L$-functions associated to these motives correspond to Hecke characters. We obtain finally an analog for these motives of a result of 
Deuring for CM elliptic curves.

Let $p$ be an odd prime, fixed once and for all, such that $E$ has good reduction for all primes over $p$. Let $S^{\prime}$ be the set of places that divide the conductor of the elliptic curve $\mathfrak{f}$ (that are the same places where $E$ has bad reduction) and the places that divide $p$.

Let $\varphi: I_{K} \rightarrow K^{*}$ be the $\mathrm{CM}$ character associated to the elliptic curve $E$ where $I_{K}$ is the idèles of $K$. Denote by $T_{w}:=\otimes_{\mathbb{Q}}^{w} K$ with $w$ a positive integer. Observe that $T_{w}$ is equal to a product of fields $\prod_{\theta} T_{\theta}$, where $\theta$ runs through the $\operatorname{Aut}(\mathbb{C})$-orbits of $\beth=\operatorname{Hom}\left(T_{w}, \mathbb{C}\right), \theta \subseteq \mathcal{I}$. Let $e_{\theta}$ be the idempotent corresponding to $T_{\theta}$ of $T_{w}$.

Define the CM character

$$
\underline{\psi}_{\theta}: I_{K} \rightarrow T_{\theta}^{*}
$$

by $\underline{\psi}_{\theta}=e_{\theta} \cdot\left(\otimes^{w} \underline{\varphi}\right)$, and denote by $\mathfrak{f}_{\theta}$ the conductor of $\underline{\psi}_{\theta}$. Observe that $\mathfrak{f}_{\theta} \mid \mathfrak{f}$ since $\underline{\psi}_{\theta}$ is a sub-representation of $\otimes^{w} \underline{\varphi}$.

Let us fix once and for all an embedding $K \rightarrow \mathbb{C}$ like in the last paragraph on [8, p.132].

We have a natural embedding $¥$

$$
K \rightarrow \otimes{ }^{w} K \rightarrow T_{w} \rightarrow T_{\theta}
$$

where the first map corresponds to the diagonal map.

For any $\vartheta \in \theta$ which its orbit in $\Xi$ is equal to $\theta$, we have a map $\vartheta: T_{w} \rightarrow$ $\vartheta\left(T_{w}\right) \subseteq \mathbb{C}$ inducing an isomorphism $T_{\theta}=T_{w} / \operatorname{ker}(\vartheta) \rightarrow \vartheta\left(T_{w}\right)$, and $\vartheta\left(T_{w}\right)$ is the field generated by $\lambda_{1}(K) \cdot \ldots \cdot \lambda_{w}(K)$ where $\lambda_{i} \in \operatorname{Hom}(K, \mathbb{C})$, which is isomorphic to $K$.

Define by $\theta_{K}$ the subset of $\theta$ which contains the $\vartheta \in \theta$ which orbit is equal to $\theta$ such that $T_{\theta}=T_{w} / \operatorname{ker}(\vartheta) \rightarrow \vartheta\left(T_{w}\right) \subset \mathbb{C}$ is in $\operatorname{Hom}_{K}\left(T_{\theta}, \mathbb{C}\right)$ for the natural embedding $¥$. In our case $\theta_{K}$ contains only one element. Let be $\vartheta=$ $\left(\lambda_{1}, \ldots, \lambda_{w}\right) \in \mathbf{J}$, we set $a_{\vartheta}=\#\left\{i \mid \lambda_{i} \in \operatorname{Hom}_{K}(K, \mathbb{C})\right\}$ and $b_{\vartheta}=w-a_{\vartheta}$. The infinite type of $\psi_{\theta}$ is defined by $\left(a_{\theta}, b_{\theta}\right):=\left(a_{\vartheta}, b_{\vartheta}\right)$ where $\vartheta$ is the element in $\theta_{K}$. Observe that $\theta$ only contains two elements, the element of $\theta$ different for $\vartheta=\left(\lambda_{1}, \ldots, \lambda_{w}\right) \in \theta_{K}$ is $\left(\overline{\lambda_{1}}, \ldots, \overline{\lambda_{w}}\right)$ where $\overline{\lambda_{i}}$ denotes the composition of $\lambda_{i}$ with the complex conjugation.

Consider the category of Chow motives $\mathcal{M}(K)$ over $K$ with morphisms induced by graded correspondences in Chow theory. We have then a natural covariant functor $h$ from the category of smooth and projective varieties over $K$ to $\mathcal{M}(K)$. Then the motive $h(E)$ of an elliptic curve $E$ over $K$ has a decomposition with respect to the zero section $h(E) \cong h^{0} E \oplus h^{1} E \oplus h^{2} E$, where $h^{0} E=h(\operatorname{Spec}(K))$ and $h^{2} E=h(\operatorname{Spec}(K))(-1)$. We can also consider the category $\mathcal{M}_{\mathbb{Q}}(K)$ which consist of the same objects but tensoring by $\mathbb{Q}$ the group of morphisms.

The motive $h^{1} E$ has multiplication by $\mathcal{O}_{K}$. Consider then the motive $\otimes^{w} h^{1} E$, has multiplication by $\mathcal{O}_{w}:=\otimes_{\mathbb{Z}}^{w} \mathcal{O}_{K}$. Then $\otimes^{w} h^{1} E_{\mathbb{Q}}$ has multiplication by $T_{w}$. Notice that $e_{\theta}$ is not integral in general for $w>1$, but is contained in $\mathcal{O}_{K}\left[1 / D_{K}\right]$. Let's denote by

$$
M_{\theta}:=e_{\theta}\left(\otimes^{w} h^{1}(E)_{\mathbb{Q}} \otimes_{\mathcal{O}_{K}} \mathcal{O}_{K}\left[1 / D_{K}\right]\right),
$$

considered as an motive with coefficients in $\mathcal{O}_{K}\left[1 / D_{K}\right]$, and by $M_{\theta \mathbb{Q}}$ its image in $\mathcal{M}_{\mathbb{Q}}(K)$. As $\lambda_{i} \in\{\lambda, \bar{\lambda}\}$ where $\lambda$ is the fixed embedding of $K$ in $\mathbb{C}$, and 
$\lambda_{i}\left(\mathcal{O}_{K}\right)=\mathcal{O}_{K}$, we have then that $e_{\theta}\left(\otimes h^{1}(E) \otimes_{\mathcal{O}_{K}} \mathcal{O}_{K}\left[1 / D_{K}\right]\right) \in \mathcal{M}(K)$ has multiplication by $\mathcal{O}_{\theta}:=e_{\theta}\left(\mathcal{O}_{w} \otimes_{\mathcal{O}_{K}} \mathcal{O}_{K}\left[1 / D_{K}\right]\right)$, and $M_{\theta \mathbb{Q}}$ has multiplication by $T_{\theta} \cong K$.

Our objective in this section is to study the $p$-adic and Betti realizations of this motive $M_{\theta}$, called Hecke motive, and to determine its $L$ function.

There are at least three equivalent notions of a Hecke character, see [12, p.48]. One is the notion of CM-character used above, [12, p.48, definition 2]. For Hecke $L$-functions and the Galois group action on the $p$-adic realization associated to the Hecke motive, we use the notion of a character which is trivial on $K^{*}$ and with image in some idèle group, $\psi_{\theta}: I_{K} / K^{*} \rightarrow I_{T_{\theta}}$ [12, p.48, definition 3]. The associated complex Hecke character, in order to define the Hecke $L$-function, is constructed from $\psi_{\theta}$ by taking the archimedian places of $I_{T_{\theta}}$ which correspond to the fixed immersion of $K$ in $\mathbb{C}$ in our situation, which we also call $\psi_{\theta}$. The character constructed from $\psi_{\theta}$ by taking the components of the places of $I_{T_{\theta}}$ above $p$ is called $\psi_{\theta, p}$ and is related with the Galois action on the $p$-adic realization associated to the motive. The character $\psi_{\theta, p}$ factors through $\operatorname{Gal}\left(K^{a b} / K\right)$ and has image in $\left(T_{\theta} \otimes \mathbb{Z}_{p}\right)^{*}$. We will use the term Hecke character when we want to consider this second notion from now on. The third notion [12, definition 1,p.48], corresponds to certain map $\widetilde{\psi}_{\theta}: \mathcal{I}_{\mathfrak{f}_{\theta}} \rightarrow T_{\theta}^{*}$, where $\mathcal{I}_{\mathfrak{f}_{\theta}}$ is the ideal classes of $K$ prime to $\mathfrak{f}_{\theta}$. So, if $\mathfrak{p}$ is a prime ideal of $\mathcal{O}_{K}$ prime to $\mathfrak{f}_{\theta}$, we mean for $\psi_{\theta}(\mathfrak{p})$ or $\underline{\psi}_{\theta}(\mathfrak{p})$ the value of the Hecke character or CM character at the idèle which has an uniformizer $\pi$ at the place $\mathfrak{p}$ and 1 in the other places. We have $\psi_{\theta}(\mathfrak{p})=\psi_{\theta}(\mathfrak{p})=\widetilde{\psi}_{\theta}(\mathfrak{p})$ (see [12, p.49-50]).

The $p$-adic realization of the motive $M_{\theta \mathbb{Q}}(w)$ is, by definition, $H_{e t}^{w}\left(M_{\theta \mathbb{Q}} \times_{K}\right.$ $\left.\bar{K}, \mathbb{Q}_{p}(w)\right)$ and we denote it by $M_{\theta \mathbb{Q}_{p}}(w)$.

Lemma 2.1. Let $p$ be a prime such that $p \nmid D_{K}$. The integral $p$-adic realization of $M_{\theta}(w), H_{e t}^{w}\left(M_{\theta} \times_{K} \bar{K}, \mathbb{Z}_{p}(w)\right) \otimes_{\mathcal{O}_{K}} \mathcal{O}_{K}\left[1 / D_{K}\right]$, is isomorphic to

$$
e_{\theta}\left(\otimes^{w} T_{p} E\right)
$$

as free $e_{\theta}\left(\otimes^{w} \mathcal{O}_{K}\left[1 / D_{K}\right] \otimes \mathbb{Z}_{p}\right)$-modules of rank 1 , with $\operatorname{Gal}(\bar{K} / K)$-action on $e_{\theta}\left(\otimes^{w} T_{p} E\right)$ given by the Hecke character $\bar{\psi}_{\theta, p}$.

Proof. Observe first that $T_{p} E$ is isomorphic as Galois modules to $H_{e t}^{1}\left(h^{1}(E) \times_{K}\right.$ $\left.\bar{K}, \mathbb{Z}_{p}(1)\right)=\operatorname{Hom}\left(T_{p} E, \mathbb{Z}_{p}(1)\right)$ by the use of Weil pairing but the isomorphism change the action of $\mathcal{O}_{K}$ to its conjugate.

The claim that $e_{\theta}\left(\otimes^{w} T_{p} E\right)$ is a free module of rank 1 follows because $T_{p} E$ is a free $\mathcal{O}_{K} \otimes \mathbb{Z}_{p} \cong \mathcal{O}_{K}\left[1 / D_{K}\right] \otimes \mathbb{Z}_{p}$-module of rank 1 and then $e_{\theta} \cdot\left(T_{p} E \otimes \ldots \otimes T_{p} E\right)$ is a free $e_{\theta} \cdot\left(\otimes^{w}\left(\mathcal{O}_{K}\left[1 / D_{K}\right] \otimes \mathbb{Z}_{p}\right)\right)$-module of rank one.

Now, consider the natural action of $G_{K}:=\operatorname{Gal}(\bar{K} / K)$ on $H_{e t}^{1}\left(h^{1}(E) \times_{K}\right.$ $\left.\bar{K}, \mathbb{Z}_{p}(1)\right)$. Since $G_{K}$ acts on the Tate module by the Hecke character $\varphi_{p}$ : $G_{K} \rightarrow\left(\mathcal{O}_{K} \otimes \mathbb{Z}_{p}\right)^{*}$, so it acts on $H_{e t}^{1}\left(h^{1}(E) \times_{K} \bar{K}, \mathbb{Z}_{p}(1)\right)$ by $\bar{\varphi}_{p}$. Using

$$
H^{w}\left(\left(h^{1}(E) \times_{K} \bar{K}\right)^{w}, \mathbb{Z}_{p}(w)\right)=H^{1}\left(h^{1}(E) \times_{K} \bar{K}, \mathbb{Z}_{p}(1)\right)^{\otimes w}
$$

and taking our idempotent, the action is given by $e_{\theta}\left(\otimes^{w} \bar{\varphi}_{p}\right)=\bar{\psi}_{\theta, p}$.

Twisting by $l+1$ we get for $p \nmid D_{K}$ that the integral $p$-adic realization for $M_{\theta}(w+l+1)$ is isomorphic to $e_{\theta}\left(\otimes^{w} T_{p} E\right)(l+1)$ with $G_{K}$-action on $e_{\theta}\left(\otimes^{w} T_{p} E\right)(l+$ 1 ) given by $\bar{\psi}_{\theta, p}$ multiplied by the $l+1$-th power of the $p$-adic cyclotomic character. 
The Betti realization for the motive $M_{\theta \mathbb{Q}}(w+l)$ named $H_{B}^{w}\left(M_{\theta \mathbb{C}}, \mathbb{Q}(w+l)\right)$ is isomorphic to $e_{\theta}\left(\otimes^{w} H_{B}^{1}(E(\mathbb{C}), \mathbb{Q}(1))\right)(l)$, we remember that we fixed once and for all an immersion for $K \subseteq \mathbb{C}$. $E(\mathbb{C})$ is the set of closed points with the analytic topology. We have

$$
\otimes_{\mathbb{Z}}^{w}\left(H_{B}^{1}\left(E \times_{K} \mathbb{C}\right), \mathbb{Z}(1)\right) \otimes_{\mathcal{O}_{K}} \mathcal{O}_{K}\left[1 / D_{K}\right]
$$

$\mathrm{a} \otimes{ }^{w} \mathcal{O}_{K}\left[1 / D_{K}\right]$-module of rank 1 and taking the idempotent $e_{\theta}$ we obtain

$$
\left.e_{\theta}\left(\otimes^{w} H_{B}^{1}\left(E \times_{K} \mathbb{C}\right), \mathbb{Z}(1)\right) \otimes \mathcal{O}_{K} \mathcal{O}_{K}\left[1 / D_{K}\right]\right)(l),
$$

a $\mathcal{O}_{\theta}$-module of rank 1 which is the submodule of $H_{B}^{w}\left(M_{\theta \mathbb{C}}, \mathbb{Q}(w+l)\right)$ corresponding to $\left.H_{B}^{w}\left(M_{\theta \mathbb{C}}, \mathbb{Z}(w+l)\right)\right) \otimes_{\mathcal{O}_{K}} \mathcal{O}_{K}\left[1 / D_{K}\right]$.

Now, we are going to study the $L$-function that corresponds to the $p$-adic representation $M_{\theta \mathbb{Q}_{p}}=H_{e t}^{w}\left(M_{\theta \mathbb{Q}} \times_{K} \bar{K}, \mathbb{Q}_{p}\right)$ of $M_{\theta \mathbb{Q}}$.

The Tamagawa number conjecture describes conjecturally special values of the $L$-function for the motive and this $L$-function involves the product of all Euler factors (one for every non-arquimedian place), but for the $p$-part of this conjecture, Kato reformulates the conjecture in terms of the partial $L$-function avoiding a non-vanishing finite set of Euler factors, more concretely the finite set contains the Euler factors coming from the primes above $p$ and the primes where the motive has bad reduction (see [20, Proposition 7.8], or [2, Chapter 1] for an overview, and see remark 2.5 for these no-vanishing in our setting).

Let $S$ be the set of places of $K$ that divide $\mathfrak{f}_{\theta}$ or $p$. Define as usual

$$
L_{S}\left(M_{\theta \mathbb{Q}}, s\right):=\prod_{\mathfrak{l} \notin S} \operatorname{det}_{\mathbb{Q}_{p}}\left(1-\operatorname{Frob}_{\mathfrak{l}} N \mathfrak{l}^{-s} \mid M_{\theta \mathbb{Q}_{p}}^{I_{\mathfrak{l}}}\right)^{-1}
$$

where $F_{r o b}$ means the geometric Frobenius, $I_{\mathfrak{l}}$ the inertia group at $\mathfrak{l}$ and $N \mathfrak{l}$ the norm $N_{K / \mathbb{Q}}$ l.

Our goal is to compute this determinant and to relate it to the local factors of the $L$-function of the Hecke character $\psi_{\theta}$ that is defined by

$$
L_{S}\left(\psi_{\theta}, s\right):=\prod_{\mathfrak{l} \notin S}\left(1-\frac{\psi_{\theta}(\mathfrak{l})}{{N \mathfrak{l}^{s}}^{s}}\right)^{-1} .
$$

Recall that the operation of the decomposition group $D_{\mathfrak{p}}$ for $\mathfrak{p} \nmid p$ on $H_{e t}^{1}\left(h^{1}(E) \times_{K} \bar{K}, \mathbb{Q}_{p}\right)$ is given by the operation of $\left.\varphi^{-1}\right|_{K_{\mathfrak{p}}^{*}}$, and hence $D_{\mathfrak{p}}$ operates on $M_{\theta \mathbb{Q}_{p}}$ via $\psi_{\theta}^{-1}$. On one hand, the inertia group $I_{\mathfrak{p}}$ acts non-trivially if and only if $\mathfrak{p}$ divides the conductor $\mathfrak{f}_{\theta}$. On the other hand, for $\mathfrak{p} \nmid \mathfrak{f}_{\theta}$, the geometric Frobenius $F r_{\mathfrak{p}}$ at $\mathfrak{p}$ acts via $\psi_{\theta}(\mathfrak{p})$. We obtain hence the following result.

Lemma 2.2 (Deninger, prop. 1.3.2.a 8]). Let $\mathfrak{l}$ be a finite prime of $K$ with $\mathfrak{l} \nmid p \mathfrak{f}_{\text {theta }}$, where $\mathfrak{f}_{\theta}$ is the conductor of the Hecke character $\psi_{\theta}$. Then

$$
\operatorname{det}_{T_{\theta} \otimes \mathbb{Q}_{p}}\left(1-F r_{\mathfrak{l}} N \mathfrak{l}^{-s} \mid M_{\theta \mathbb{Q}_{p}}^{I_{\mathfrak{l}}}\right)=\left(1-\psi_{\theta}(\mathfrak{l}) N \mathfrak{l}^{-s}\right) .
$$

We impose some restrictions for our motive $M_{\theta}(w+l+1)$ once and for all. We suppose $-w-2 l \leq-3$. Remember that, with the restriction that $E$ is defined over $K$, we have $\#|\theta|=2$, and, in particular, we have $T_{\theta} \cong K$ and for $p \nmid D_{K}$ we have $\mathcal{O}_{\theta} \otimes \mathbb{Z}_{p} \cong \mathcal{O}_{K} \otimes \mathbb{Z}_{p}$.

The $L$-function for $M_{\theta \mathbb{Q}}$ can be described by using lemma 2.2 and by taking the norm map. 
Lemma 2.3. Let $\mathfrak{l}$ a prime of $K$ such that $\mathfrak{l} \nmid \mathfrak{f}_{\theta}$ and it is prime to $p$. We have then the following equality

$$
\operatorname{det}_{\mathbb{Q}_{p}}\left(1-F r_{\mathfrak{l}} N \mathfrak{l}^{-s} \mid M_{\theta \mathbb{Q}_{p}}^{I_{\mathfrak{l}}}\right)=\left(1-\psi_{\theta}(\mathfrak{l}) N \mathfrak{l}^{-s}\right)\left(1-\overline{\psi_{\theta}}(\mathfrak{l}) N \mathfrak{l}^{-s}\right) .
$$

As a corollary we obtain a generalization of a result of Deuring.

Theorem 2.4. Let $S$ be the set of the primes on $K$ dividing $\mathfrak{f}_{\theta}$ and primes dividing $p$. Then:

$$
L_{S}\left(M_{\theta \mathbb{Q}}, s\right)=L_{S}\left(\psi_{\theta}, s\right) L_{S}\left(\bar{\psi}_{\theta}, s\right) .
$$

Remark 2.5. The p-adic realization $V_{p}:=M_{\theta \mathbb{Q}_{p}}(w+l+1)$ satisfies that the local Euler factors

$$
P_{\mathfrak{p}}\left(V_{p}^{*}(1), 0\right)=P_{\mathfrak{p}}\left(\bar{\psi}_{\theta},-l\right)
$$

are different from 0 for all $\mathfrak{p} \in S$ where $V_{p}^{*}$ is the dual Galois module of $V_{p}$. Hence, it satisfies the hypothesis of [19, conjecture 2.2.7].

To show this fact, suppose first that $\mathfrak{p} \mid \mathfrak{f}_{\theta}$. Then the inertia group acts nontrivially on $V_{p}$, which is a one dimensional $\mathcal{O}_{\theta} \otimes \mathbb{Q}$-module, and hence

$$
L_{\mathfrak{p}}\left(\bar{\psi}_{\theta}, s\right)=1
$$

for all $\mathfrak{p} \mid \mathfrak{f}_{\theta}$, and in particular for $s=-l$.

If $\mathfrak{p}$ divides $p$, then the result follows from the fact that any proper smooth variety with good reduction at $\mathfrak{p}$ satisfies it for weight reasons, and in particular:

$$
\operatorname{det}_{\mathbb{Q}_{p}}\left(1-F r_{\mathfrak{p}} N \mathfrak{p}^{l} \mid H_{e t}^{w}\left(\left(E \times_{K} \bar{K}\right)^{w}, \mathbb{Q}_{l^{\prime}}\right)\right) \neq 0,
$$

with $l^{\prime} \neq p$, and therefore, since the different idempotents $e_{\theta}$ give a direct summand of the cohomology group $H^{w}\left(\left(E \times_{K} \bar{K}\right)^{w}, \mathbb{Q}_{l^{\prime}}\right)$,

$$
L_{\mathfrak{p}}\left(\bar{\psi}_{\theta},-l\right) \neq 0 .
$$

The motivic cohomology group $H_{\mathcal{M}}^{w+1}\left(M_{\theta \mathbb{Q}}, \mathbb{Q}(w+l+1)\right)$ is the $K$-theory group $K_{2(w+l)-w+1}\left(M_{\theta \mathbb{Q}}\right)^{(w+w+1)} \otimes \mathbb{Q}$ where the $K$-groups are the Quillen $K$ groups and the superscript denotes the Adam's filtration on them.

We suppose recall that $w-2(w+l+1) \leq-3$. We have a Beilinson regulator map,

$$
r_{\mathcal{D}}: H_{\mathcal{M}}^{w+1}\left(M_{\theta \mathbb{Q}}, \mathbb{Q}(w+l+1)\right) \rightarrow H_{B}^{w}\left(M_{\theta \mathbb{C}}, \mathbb{Q}(w+l)\right) \otimes_{\mathbb{Q}} \mathbb{R},
$$

and the Soulé regulator map:

$$
r_{p}: H_{\mathcal{M}}^{w+1}\left(M_{\theta \mathbb{Q}}, \mathbb{Q}(w+l+1)\right) \rightarrow H_{e ̂ t}^{1}\left(\mathcal{O}_{K}[1 / S], M_{\theta \mathbb{Q}_{p}}(w+l+1)\right) .
$$

The $p$-part of the Tamagawa number conjecture claims in particular that $r_{\mathcal{D}} \otimes_{\mathbb{Q}} \mathbb{R}$ and $r_{p} \otimes_{\mathbb{Q}} \mathbb{Q}_{p}$ are isomorphisms. Deninger in $8 / 7$ constructs a $\mathbb{Q}$ subspace $H_{\mathcal{M}}^{\text {constr }}$ of $H_{\mathcal{M}}^{w+1}\left(M_{\theta \mathbb{Q}}, \mathbb{Q}(w+l+1)\right)$ such that $r_{\mathcal{D}} \otimes \mathbb{R}$ restricted to $H_{\mathcal{M}}^{\text {constr }} \otimes_{\mathbb{Q}} \mathbb{R}$ is an isomorphism. The term weak in the formulation of the $p$-part of the Tamagawa number conjecture means that this conjecture is proved using the space $H_{\mathcal{M}}^{\text {constr }}$ instead of $H_{\mathcal{M}}^{w+1}\left(M_{\theta \mathbb{Q}}, \mathbb{Q}(w+l+1)\right)$. 


\section{The Beilinson conjecture for Hecke characters}

In this section we review the work on the Beilinson conjecture for the motive $M_{\theta \mathbb{Q}}(w+l+1)$ done by Deninger in [8], under the language of the Tamagawa number conjecture.

Theorem 3.1 (Deninger, Theorem 1.4.1 [8]). Let $w=a_{\theta}+b_{\theta} \geq 1$. Consider an integer $l$ such that

$$
\begin{gathered}
-l \leq \operatorname{Min}\left(a_{\theta}, b_{\theta}\right) \quad \text { if } a_{\theta} \neq b_{\theta} \\
-l<a_{\theta}=b_{\theta}=w / 2 \quad \text { otherwise. }
\end{gathered}
$$

Then the L-series $L\left(\bar{\psi}_{\theta}, s\right)$ has a zero of order 1 at $s=-l$, (i.e. $\operatorname{ord}_{s=-l} L\left(\bar{\psi}_{\theta}, s\right)=$ 1).

Moreover, there exist an element $\xi_{\theta}$ in $H_{\mathcal{M}}^{w+1}\left(M_{\theta \mathbb{Q}}, \mathbb{Q}(w+l+1)\right)$, such that

$$
r_{\mathcal{D}}\left(\xi_{\theta}\right)=\lim _{s \rightarrow-l} \frac{L\left(\overline{\psi_{\theta}}, s\right)}{s+l} \eta_{\theta} \bmod T_{\theta}^{*}
$$

in the free rank one $T_{\theta} \otimes \mathbb{R}$-module $H_{B}^{w}\left(M_{\theta \mathbb{C}}, \mathbb{R}(w+l)\right)$, where $\eta_{\theta}$ is a $T_{\theta}$-generator of $H_{B}^{w}\left(M_{\theta \mathbb{C}}, \mathbb{Q}(w+l)\right)$.

Let's recall the construction of $\xi_{\theta}$, following the results of Deninger. We suppose once for all that $l \geq 0$.

Fix an algebraic differential form $\omega \in H^{0}\left(E, \Omega_{E / K}\right)$. Since we have complex multiplication, we can write the period lattice as $\Gamma=\Omega \mathcal{O}_{K}$, where $\Omega \in \mathbb{C}^{*}$ is the complex period. Fix an element $\gamma$ in $H_{1}(E(\mathbb{C}), \mathbb{Z})$ such that it is an $\mathcal{O}_{K}$-generator, and satisfies

$$
\Omega=\int_{\gamma} \omega
$$

By Poincaré duality, we have that $\gamma$ corresponds to $\eta_{\gamma}$, an $\mathcal{O}_{K}$-generator for $H^{1}(E(\mathbb{C}), \mathbb{Z}(1))$. Thus $\eta_{\gamma} \otimes_{\mathcal{O}_{K}} \mathcal{O}_{K}\left[1 / D_{K}\right]$ is an $\mathcal{O}_{K}\left[1 / D_{K}\right]$-generator for the module $H^{1}(E(\mathbb{C}), \mathbb{Z}(1)) \otimes_{\mathcal{O}_{K}} \mathcal{O}_{K}\left[1 / D_{K}\right]$ which by abuse of notation we call also $\eta_{\gamma}$. Consider now the $\mathcal{O}_{\theta}$-generator

$$
\eta_{\theta}:=(2 \pi i)^{l} e_{\theta}\left(\otimes^{w} \eta_{\gamma}\right)
$$

of $H_{B}^{w}\left(M_{\theta \mathbb{C}}, \mathbb{Z}(w+l)\right) \otimes_{\mathcal{O}_{K}} \mathcal{O}_{K}\left[1 / D_{K}\right]$.

To construct $\xi_{\theta}$, we will define a divisor on the torsion points of the elliptic curve; its image by the composition of the Eisenstein map $\mathcal{E}_{\mathcal{M}}([7, \S 8])$ with the Deninger projector map $\mathcal{K}_{\mathcal{M}}([8,(2.8)])$ will define our $\xi_{\theta}$.

Remember that $\mathfrak{f}_{\theta}$ is the conductor of the Hecke character $\psi_{\theta}$ associated with $M_{\theta}$, and denote by $f$ a generator of $\mathfrak{f}_{\theta}$ (it exists since $c l(K)=1$ ). We have that

$$
\Omega f^{-1} \in \mathfrak{f}_{\theta}^{-1} \Gamma
$$

and that $\left(\Omega f^{-1}\right)$ gives a divisor in $\mathbb{Z}\left[E\left[\mathfrak{f}_{\theta}\right] \backslash 0\right]$ defined over $K\left(E\left[\mathfrak{f}_{\theta}\right]\right)$. Since $\mathfrak{f}$ is the conductor of $\psi$ and $\mathfrak{f}_{\theta} \mid \mathfrak{f}$, the divisor $\left(\Omega f^{-1}\right)$ is defined also over $K(E[\mathfrak{f}])$. We will define our divisor as

$$
\beta_{\theta}:=N_{K(E[\mathfrak{f}]) / K}\left(\left(\Omega f^{-1}\right)\right) .
$$


Denote by $\rho_{\theta}$ a finite idèle such that $\left(\rho_{\theta}\right)=\mathfrak{f}_{\theta}$ and $v_{\mathfrak{p}}\left(f^{-1}-\rho_{\mathfrak{p}}^{-1}\right) \geq 0$ for all $\mathfrak{p} \mid \mathfrak{f}_{\theta}$.

If $a_{\theta} \not \equiv b_{\theta} \bmod \left|\mathcal{O}_{K}^{*}\right|$, we obtain that ([8, p.142,(2.11)])

$$
r_{\mathcal{D}}\left(\mathcal{K}_{\mathcal{M}} \mathcal{E}_{\mathcal{M}}\left(\beta_{\theta}\right)\right)=(-1)^{l-1} \frac{2^{l-1} N_{K / \mathbb{Q}} \mathfrak{f}_{\theta}^{w+2 l} \psi_{\theta}\left(\rho_{\theta}\right)}{(2 l+w) ! N_{K / \mathbb{Q}}\left(\mathfrak{f}_{\theta}\right)^{l+w}} \frac{\Phi(\mathfrak{f})}{\Phi\left(\mathfrak{f}_{\theta}\right)} L^{\prime}\left(\overline{\psi_{\theta}},-l\right) \eta_{\theta}
$$

where $\Phi(\mathfrak{m}):=\left|\left(\mathcal{O}_{K} / \mathfrak{m}\right)^{*}\right|$ for any ideal $\mathfrak{m}$ of $\mathcal{O}_{K}$.

This is an analog for $M_{\theta}(w+l+1)$ of [22, thm.1.2.2], which corresponds to the case $h^{1}(E)(1+l+1)$.

Theorem 3.2 (Deninger, $\S 2[8])$. Suppose that $a_{\theta} \not \equiv b_{\theta} \bmod \left(\# \mathcal{O}_{K}^{*}\right)$ and that $a_{\theta}, b_{\theta}, l$ satisfy the hypothesis of the theorem 3.1 with $l \geq 0$. Define, by using the previous notation,

$$
\begin{gathered}
\xi_{\theta, l}:= \\
(-1)^{l-1} \frac{(2 l+w) ! L_{p}\left(\overline{\psi_{\theta}},-l\right)^{-1} \Phi\left(\mathfrak{f}_{\theta}\right)}{2^{l-1} N_{K / \mathbb{Q}} \mathfrak{f}_{\theta}^{l} \psi_{\theta}\left(\rho_{\theta}\right) \Phi(\mathfrak{f})} \mathcal{K}_{\mathcal{M}} \circ \mathcal{E}_{\mathcal{M}}^{2 l+w}\left(\beta_{\theta}\right) \in H_{\mathcal{M}}^{w+1}\left(M_{\theta \mathbb{Q}}, \mathbb{Q}(w+l+1)\right),
\end{gathered}
$$

where $L_{p}\left(\overline{\psi_{\theta}}, s\right)$ is the product of the Euler factors for the primes of $K$ above $p$.

Then

$$
r_{\mathcal{D}}\left(\xi_{\theta, l}\right)=L_{S}^{*}\left(\overline{\psi_{\theta}},-l\right) e_{\theta}\left(\otimes^{w} \eta_{\gamma}\right),
$$

where $S$ are the primes of $K$ that divide $\mathfrak{f}_{\theta} p$. Here $L_{S}^{*}\left(\overline{\psi_{\theta}},-l\right)=\lim _{s+l \rightarrow 0} L_{S}\left(\overline{\psi_{\theta}}, s\right) /(s+$ l).

Definition 3.3. For $a_{\theta} \not \equiv b_{\theta} \bmod \left(\# \mathcal{O}_{K}^{*}\right)$ we define

$$
\mathcal{R}_{\theta}:=\xi_{\theta, l} \mathcal{O}_{K} .
$$

Remark 3.4. Theorem 1.4.1[8] is more general because it includes the situation $a_{\theta} \equiv b_{\theta} \bmod \left(\# \mathcal{O}_{K}^{*}\right)$. But in this situation, Deninger defines a divisor $\tilde{\beta}_{\theta}$ instead of $\beta_{\theta}$ which is not a norm of a positive divisor and moreover it contains the zero point of E. Thus the techniques for constructing an Euler system of $\$ 5$ can not be applied in this case (see for example Theorem 5.1).

As a consequence of Theorem 3.2, we have that our submodule $\mathcal{R}_{\theta}$ verifies some integral version of the Beilinson conjecture for the motive $M_{\theta}(w+l+1)$.

Theorem 3.5. The $\mathcal{O}_{K}$-submodule $\mathcal{R}_{\theta}$ of $H_{\mathcal{M}}^{w+1}\left(M_{\theta \mathbb{Q}}, \mathbb{Q}(w+l+1)\right)$ satisfies that

$$
\begin{aligned}
& \operatorname{det}_{\mathcal{O}_{K}\left[1 / D_{K}\right]}\left(r_{\mathcal{D}}\left(\mathcal{R}_{\theta} \otimes_{\mathcal{O}_{K}} \mathcal{O}_{K}\left[1 / D_{K}\right]\right)\right)= \\
& L_{S}^{*}\left(\overline{\psi_{\theta}},-l\right) \operatorname{det}_{\mathcal{O}_{K}\left[1 / D_{K}\right]}\left(H_{B}^{w}\left(M_{\theta}, \mathbb{Z}(w+l)\right) \otimes_{\mathcal{O}_{K}} \mathcal{O}_{K}\left[1 / D_{K}\right]\right) \\
& \text { in } \operatorname{det}_{\mathcal{O}_{K}\left[1 / D_{K}\right] \otimes \mathbb{R}}\left(\left(H_{B}^{w}\left(M_{\theta \mathbb{C}}, \mathbb{Z}(w+l)\right) \otimes_{\mathcal{O}_{K}} \mathcal{O}_{K}\left[1 / D_{K}\right]\right) \otimes \mathbb{R}\right) \text {. }
\end{aligned}
$$

Proof. Observing that $\eta_{\theta}$ is a $\mathcal{O}_{\theta}$-base for the free $\mathcal{O}_{\theta}$-module

$$
H_{B}^{w}\left(M_{\theta \mathbb{Q}} \times_{K} \mathbb{C}, \mathbb{Z}(w+l)\right) \otimes_{\mathcal{O}_{K}} \mathcal{O}_{K}\left[1 / D_{K}\right]
$$

of rank one, the result follows. 
Corollary 3.6. The submodule $\mathcal{R}_{\theta}$ defined above satisfies the Beilinson conjecture inside the p-part of the Tamagawa number conjecture for $p \nmid D_{K}$, that is $\mathcal{R}_{\theta}$ satisfies the following conditions:

1. The map $r_{\mathcal{D}} \otimes \mathbb{R}$ is a isomorphism when restricted to $\mathcal{R}_{\theta} \otimes \mathbb{R}$.

2. $\operatorname{dim}_{\mathbb{Q}}\left(H_{B}^{w}\left(M_{\theta \mathbb{C}}, \mathbb{Q}(w+l)\right)\right)=\operatorname{ord}_{s=-l} L_{S}\left(M_{\theta \mathbb{Q}}, s\right)=2$.

3. We have the following equality

$$
\begin{gathered}
r_{\mathcal{D}}\left(\operatorname{det}_{\mathbb{Z}\left[1 / D_{K}\right]}\left(\mathcal{R}_{\theta} \otimes_{\mathcal{O}_{K}} \mathcal{O}_{K}\left[1 / D_{K}\right]\right)\right)= \\
L_{S}^{*}\left(M_{\theta \mathbb{Q}},-l\right) \operatorname{det}_{\mathbb{Z}\left[1 / D_{K}\right]}\left(H_{B}^{w}\left(M_{\theta}, \mathbb{Z}(w+l)\right) \otimes_{\mathcal{O}_{K}} \mathcal{O}_{K}[1 / D]\right)
\end{gathered}
$$

where $L_{S}^{*}\left(M_{\theta \mathbb{Q}},-l\right)$ means $\lim _{s \rightarrow-l} L_{S}^{*}\left(M_{\theta \mathbb{Q}}, s\right) /(s+l)^{2}$ (this makes sense by using theorem 2.4 and theorem 3.1).

Proof. The first and the second conditions are clear for the dimensions of the spaces involved in the Deligne regulator map, and the theorem 3.5. The third condition comes from the previous theorem using the fact that, if we multiply an $\mathcal{O}_{\theta}$-module with an element $L_{S}^{*}\left(\overline{\psi_{\theta}},-l\right)$ in $\mathcal{O}_{\theta} \otimes \mathbb{R}$, the determinant is multiplied by the norm

$$
N_{\mathcal{O}_{\theta} \otimes \mathbb{R} / \mathbb{R}}\left(L_{S}^{*}\left(\overline{\psi_{\theta}},-l\right)\right)=L_{S}^{*}\left(\overline{\psi_{\theta}},-l\right) \overline{L_{S}^{*}\left(\overline{\psi_{\theta}},-l\right)}=L_{S}^{*}\left(\overline{\psi_{\theta}},-l\right) L_{S}^{*}\left(\psi_{\theta},-l\right) .
$$

Using theorem 2.4, we obtain that this is equal to $L_{S}^{*}\left(M_{\theta \mathbb{Q}},-l\right)$.

\section{Iwasawa theory}

We suppose once and for all that $p \nmid \# \mathcal{O}_{K}^{*}$ and $p \nmid N_{K / \mathbb{Q}}(\mathfrak{f})$, (in particular $\left.p \nmid D_{K}\right)$.

To simplify the notation, we will denote in the following by

$$
M_{\theta \mathbb{Z}_{p}}(w+l+1)=e_{\theta}\left(\otimes^{w}\left(H_{e t}^{1}\left(E \times_{K} \bar{K}, \mathbb{Z}_{p}(1)\right)\right)(l+1)\right.
$$

the $p$-adic lattice for the $p$-adic realization of $M_{\theta}(w+l+1)$.

Let $K_{n}:=K\left(E\left[p^{n+1}\right]\right)$ be the field of definition of the $p^{n+1}$-torsion points of $E, \mathcal{O}_{n}$ its ring of integers and let $K_{\infty}:=\lim K_{n}$ be its direct limit. Denote by $\mathcal{O}_{n}$ the ring of integers of $K_{n}$ (respectively $\left.\overrightarrow{\mathcal{O}}_{\infty}\right)$. We know that $\Delta:=\operatorname{Gal}\left(K_{0} / K\right)$ has order prime to $p$ and $\Gamma:=\operatorname{Gal}\left(K_{\infty} / K_{0}\right)$ is isomorphic to $\mathbb{Z}_{p}^{2}$.

Let $\mathcal{G}$ be the Galois group $\operatorname{Gal}\left(K_{\infty} / K\right)$; then $\mathcal{G} \cong \Delta \times \Gamma$.

We use now the notations on Iwasawa theory for imaginary quadratic fields used in [22, §2.1] but with a different definition of elliptic units.

Let us define the elliptic units $\mathcal{C}_{n, \mathfrak{f}_{\theta}}$ in $K_{n}$ which are more convenient for us.

For every ideal $\mathfrak{a}$ of $K$ prime to 6 we can define a theta function

$$
\theta_{\mathfrak{a}}: E \backslash \operatorname{ker}([\mathfrak{a}]) \longrightarrow \mathbb{C}
$$

which has divisor $N(\mathfrak{a})(e)-\operatorname{ker}([\mathfrak{a}])$ (for the precise definition see [22, Theorem 4.2.2]). The function $\theta_{\mathfrak{a}}(z)$ is in fact a 12-th root of the function defined in [9, II.2.4]. Let $\mathfrak{g}$ be a fixed ideal of $\mathcal{O}_{K}$ such that $\mathcal{O}_{K}^{*} \rightarrow\left(\mathcal{O}_{K} / \mathfrak{g}\right)^{*}$ is injective, and suppose that $\mathfrak{g}$ divides the conductor $\mathfrak{f}$ of the elliptic curve $E$. Let's denote by $t_{\mathfrak{g}}$ a generator for the $E[\mathfrak{g}]$-torsion points as $\mathcal{O}_{K}$-module, and let $\mathfrak{a}$ be an ideal prime to $6 \mathfrak{g}$. 
Definition 4.1. Let $C_{n, \mathfrak{g}}$ be the subgroup of units generated over $\mathbb{Z}\left[\operatorname{Gal}\left(K_{n} / K\right)\right]$ by

$$
\prod_{\sigma \in \operatorname{Gal}(K(\mathfrak{g}) / K)} \theta_{\mathfrak{a}}\left(t_{\mathfrak{g}}^{\sigma}+h_{n}\right),
$$

where $\mathfrak{a}$ runs through all ideals prime to $6 p \mathfrak{f}, K(\mathfrak{g})$ is the ray class field defined by $\mathfrak{g}$ and $h_{n}$ is a primitive $p^{n+1}$-torsion point (i.e. a generator of the $p^{n+1}$-torsion points of $E$ as $\mathcal{O}_{K}$-module). Define the group of elliptic units of $K_{n}$ as

$$
\mathcal{C}_{n, \mathfrak{g}}:=\mu_{\infty}\left(K_{n}\right) C_{n, \mathfrak{g}}
$$

where $\mu_{\infty}\left(K_{n}\right)$ denotes the roots of unity in $K_{n}$.

Denote by $\overline{\mathcal{C}}_{n, \mathfrak{g}}$ the closure in the local units $\mathcal{U}_{n}^{\mathfrak{p}}$ where $\mathcal{U}_{n}^{\mathfrak{p}}$ is the group of local units of $K_{n} \otimes_{K} K_{\mathfrak{p}}$ which are congruent to 1 modulo the primes above $\mathfrak{p}$ where $\mathfrak{p}$ is a prime of $K$ above $p$. Define $\overline{\mathcal{C}}_{\infty, \mathfrak{g}}:=\lim _{\leftarrow} \overline{\mathcal{C}}_{n, \mathfrak{g}}$, and $\mathcal{U}_{\infty}^{\mathfrak{p}}:=\lim _{\leftarrow} \mathcal{U}_{n}^{\mathfrak{p}}$

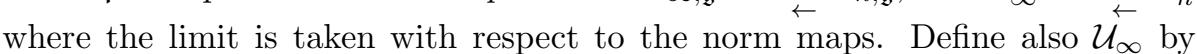
$\mathcal{U}_{\infty}^{\mathfrak{p}} \times \mathcal{U}_{\infty}^{\mathfrak{p}^{*}}$ if $p=\mathfrak{p p}^{*}$ splits, and if $p$ inert or ramified by $\mathcal{U}_{\infty}^{\mathfrak{p}}$. Let $\mathcal{Y}_{n}$ be the $p$-adic completion of $\left(K_{n} \otimes \mathbb{Q}_{p}\right)^{*}$ and $\mathcal{Y}_{\infty}:=\lim _{\leftarrow} \mathcal{Y}_{n}$.

Let us once and for all to specialize the elliptic units to $\mathfrak{g}=\mathfrak{f}_{\theta}$

Recall that $S$ denotes the set of primes of $K$ which divide $\mathfrak{f}_{\theta}$ or $p$, and that $S^{\prime}$ denotes the set of primes of $K$ which divide $p$ or the conductor $\mathfrak{f}$ of the elliptic curve $E$. Denote $\mathcal{O}_{S}:=\mathcal{O}_{K}[1 / S]$ and $\mathcal{O}_{p}:=\mathcal{O}_{K} \otimes \mathbb{Z}_{p}$.

We are going to define a map in the spirit of Soulé:

$$
(\text { Soul })_{p}: \overline{\mathcal{C}}_{\infty, \mathfrak{f}_{\theta}} \otimes_{\mathbb{Z}_{p}} M_{\theta \mathbb{Z}_{p}}(w+l) \rightarrow H^{1}\left(\mathcal{O}_{S}, M_{\theta \mathbb{Z}_{p}}(w+l+1)\right)
$$

observe that $M_{\theta \mathbb{Z}_{p}}(w+l)$ is unramified outside $S$, thus $H^{1}\left(\mathcal{O}_{S}, M_{\theta \mathbb{Z}_{p}}(w+l+1)\right)$ makes sense.

Using the definition of $M_{\theta \mathbb{Z}_{p}}(w)(l+1)$, we have that

$$
H^{1}\left(\mathcal{O}_{S}, M_{\theta \mathbb{Z}_{p}}(w+l+1)\right)=\lim _{\leftarrow} H^{1}\left(\mathcal{O}_{S},\left(e_{\theta} \otimes^{w} E\left[p^{r+1}\right]\right)(l+1)\right) .
$$

Define $(S o u l)_{p}$ in the following way. Given $\left(\theta_{r}\right)_{r}$ a norm compatible system of elliptic units and an element $\left(t_{r}\right)_{r}$ of $\lim \left(e_{\theta}\left(\otimes^{w} E\left[p^{r+1}\right]\right)\right)(l)$, we define

$$
(S o u l)_{p}\left(\left(\theta_{r} \otimes t_{r}\right)_{r}\right):=\left(N_{K_{r} / K}\left(\theta_{r} \otimes t_{r}\right)\right)_{r} .
$$

It is well defined: $\theta_{r} \otimes t_{r}$ is an element in

$$
\mathcal{O}_{r, S}^{*} /\left(\mathcal{O}_{r, S}^{*}\right)^{p^{r+1}} \otimes\left(e_{\theta}\left(\otimes^{w} E\left[p^{r+1}\right]\right)\right)(l) \subset H^{1}\left(\mathcal{O}_{r, S},\left(e_{\theta}\left(\otimes^{w} E\left[p^{r+1}\right]\right)\right)(l+1)\right)
$$

where $\mathcal{O}_{r, S}$ is $\mathcal{O}_{r}[1 / S]$ the ring of integers of $K_{r}$ inverting the primes above $S$, $N_{K_{r} / K}$ denotes the norm map in cohomology and by Soulé's Lemma 1.4 [26] one gets an element in $H^{1}\left(\mathcal{O}_{S}, M_{\theta \mathbb{Z}_{p}}(w+l+1)\right)$. The map $(S o u l)_{p}$ factors thought the coinvariants, denoted by $\left(\overline{\mathcal{C}}_{\infty, \mathfrak{f}_{\theta}} \otimes M_{\theta \mathbb{Z}_{p}}(w+l)\right)_{\mathcal{G}}$.

Definition 4.2. The Soule elliptic elements are the elements in the image of the map

$$
(\text { Soul })_{p}:\left(\overline{\mathcal{C}}_{\infty, \mathfrak{f}_{\theta}} \otimes M_{\theta \mathbb{Z}_{p}}(w+l)\right)_{\mathcal{G}} \rightarrow H^{1}\left(\mathcal{O}_{S}, M_{\theta \mathbb{Z}_{p}}(w+l+1)\right)
$$

where $\mathcal{G}=\operatorname{Gal}\left(K\left(E\left[p^{\infty}\right]\right) / K\right)$. 
We consider in the following the representation $\chi$ of the group $\Delta$ given by the action of $\Delta$ in $\mathrm{Hom}_{\mathcal{O}_{p}}\left(M_{\theta \mathbb{Z}_{p}}(w+l), \mathcal{O}_{p}\right)$.

We are only able to apply the techniques on Iwasawa theory of [22] for certain representations that we call good representation.

Definition 4.3. We say that such representation $\chi$ of the group $\Delta$ is a good representation if it satisfies two conditions in Iwasawa theory about isomorphism between some concrete Iwasawa modules: (A) the Iwasawa main conjecture of Rubin [22, Theorem 2.1.3] but replacing the elliptic units module there with the elliptic module units $\overline{\mathcal{C}}_{\infty, \mathfrak{f}_{\theta}}^{\chi}$ and $(B)$ from the inclusion $\mathcal{U}_{\infty} \subseteq \mathcal{Y}_{\infty}$ we get that $\mathcal{U}_{\infty}^{\chi} \cong \mathcal{Y}_{\infty}^{\chi}$ as Iwasawa modules for the Iwasawa ring $\lim _{\leftarrow} \mathbb{Z}_{p}\left[\left[G a l\left(K_{n} / K\right)\right]\right]^{\chi}$.

We observe that the elliptic units $\mathcal{C}_{\infty, \mathfrak{f}}$, which are the ones that appear in 25] and 22, satisfies the theorem of Iwasawa main conjecture of 24] for any $\Delta$-representation under the hypothesis of the theorem in 24] (personal communication of Rubin).

When $S=S^{\prime}$ we have $\mathcal{C}_{\infty, \mathfrak{f}_{\theta}}=\mathcal{C}_{\infty, f}[9$, Proposition II.2.5], therefore the Iwasawa main conjecture (condition (A)) is true from Rubin's theorem [24] 25] for $p$ splits and for $p$ inert when $\chi$ is non trivial on the decomposition group $\Delta_{\mathfrak{p}}$ of $\mathfrak{p}$ in $\Delta$.

Condition (B) is always true if $p$ splits [22, Lemma 2.1.6]. If $p$ is inert or ramified, the representation $\chi$ satisfies condition (B) if $\mathbb{Z}_{p}\left[\Delta / \Delta_{p}\right]^{\chi}=0$ (see [22, lemma 2.1.6]), moreover because $p$ is a prime over which $E$ has good reduction we have $\Delta_{p}=\Delta[22$, Lemma 2.2.9], thus condition (B) is true if $\chi$ is non-trivial.

Remark 4.4. We guess that the Iwasawa main conjecture [22, Theorem 2.1.3] is also true without the condition $S=S^{\prime}$ for our elliptic units and our character $\chi$, so such a character should be good if it just verify condition (B). In the next section we prove that $\overline{\mathcal{C}}_{\infty, \mathfrak{f}_{\theta}}^{\chi}$ is a rank 1 Iwasawa module and we construct an Euler system there. Using this and [9, Lemma III.1.10], it should be possible to prove the Iwasawa main conjecture for $\chi$ using the techniques in [25].

Lemma 4.5. Suppose that $\psi_{\theta}$ has infinite type $\left(a_{\theta}, b_{\theta}\right)$ with $a_{\theta} \not \equiv b_{\theta} \bmod \left(\# \mathcal{O}_{K}^{*}\right)$. Suppose also that $p$ splits, and that $\left(a_{\theta}-b_{\theta}, p-1\right)=1$. Then $S=S^{\prime}$.

Proof. Let $v$ be a prime of $K$ dividing $\mathfrak{f}$. Let $v_{0}$ be a prime of $K_{0}$ dividing $v$. Denote by $\Delta_{v_{0}}$ the stabilizer of $v_{0}$ in $K_{0}$. We have then that $I_{v_{0}} \subset \Delta_{v_{0}} \subset \Delta$ acts non-trivially in the Tate module $T_{p} E$ via the Hecke character $\varphi_{p}$. Hence, $I_{v_{0}}$ acts on $e_{\theta}\left(\otimes^{w} T_{p} E\right)$ via $\left.\psi_{\theta, p}\right|_{I_{v_{0}}}=\varphi_{p}^{a_{\theta}-b_{\theta}}$, as $I_{v_{0}}$ lies in the kernel of the $p$-adic cyclotomic character $(v$ is prime to $p$ ). Since $p$ splits, we have that $\# \Delta=(p-1)^{2}$, and since $\left(a_{\theta}-b_{\theta}, p-1\right)=1, \varphi_{p}^{a_{\theta}-b_{\theta}}$ acts non-trivial on $I_{v_{0}}$.

Lemma 4.6. Suppose that $p$ splits in $K$ and suppose that $p-1 \nmid a_{\theta}+l+1$ or $p-1 \nmid b_{\theta}+l+1$ or $p-1 \nmid a_{\theta}-b_{\theta}$. Then $\chi$, as $\Delta$-representation, is not the cyclotomic representation.

Proof. The character $\chi$ is equal to $\left(\bar{\psi}_{\theta} \kappa^{l}\right)^{-1}$ where $\kappa$ is the cyclotomic character. Since $p$ is split in $K$, we have that $p=\mathfrak{p p}^{*}$, with $\mathfrak{p} \neq \mathfrak{p}^{*}$. Let $\Delta_{\mathfrak{p}}$ be the Galois group $\operatorname{Gal}(K(E[\mathfrak{p}]) / K)$; it is a subgroup of the decomposition group since $\mathfrak{p}$ is totally ramified in $\Delta_{\mathfrak{p}}$. Observe that $M_{\theta \mathbb{Z}_{p}}$ has multiplication isomorphic to $\mathcal{O}_{K} \otimes \mathbb{Z}_{p}$ and, as $p$ splits, it decomposes in two idempotents. These idempotents decompose the Hecke character $\psi_{\theta, p}=\psi_{\Omega_{1}} \oplus \psi_{\Omega_{2}}$, see 12 for more details. It 
is known that $\left.\bar{\psi}_{\Omega_{1}}\right|_{\Delta_{\mathrm{p}}}=\kappa^{b_{\theta}}$ (see for example [12, §2.5]), so we get that our character is different from $\kappa$ as long as $\# \Delta_{\mathfrak{p}}=p-1 \nmid b_{\theta}+l+1$, since $\kappa$ is a generator for the character group of $\Delta_{\mathfrak{p}}$.

Using the same kind of argument for $\mathfrak{p}^{*}$ instead of $\mathfrak{p}$ we obtain a similar divisibility result but with $a_{\theta}$ instead of $b_{\theta}$. Thus, we obtain the cyclotomic character only in the case that $p-1 \mid a_{\theta}+l+1$ and $p-1 \mid b_{\theta}+l+1$.

Similar argument for $\bar{\psi}_{\Omega_{2}}$, we obtain the same simultaneous arithmetic conditions, i.e. $p-1 \mid l+b_{\theta}+1$ and $p-1 \mid a_{\theta}+l+1$ in order to obtain the cyclotomic character. We refer to [13, p.220,pp.223-234] for more details on the characters $\psi_{\Omega_{1}}$ and $\psi_{\Omega_{2}}$.

Next theorem is the analog of [22, Theorem 2.2.12] in our situation.

Theorem 4.7. Suppose that $p$ is an odd prime, prime to $N_{K / \mathbb{Q}} \mathfrak{f}$ and to $\# \mathcal{O}_{K}^{*}$. Let the $\Delta$-representation $\chi$ on $\operatorname{Hom}_{\mathcal{O}_{p}}\left(M_{\theta \mathbb{Z}_{p}}(w+l), \mathcal{O}_{p}\right)$ be a good representation. Then the map $(\text { Soul })_{p}$ induces an isomorphism of $\mathcal{O}_{p}$-modules

$\operatorname{det}_{\mathcal{O}_{p}}\left(\left(\overline{\mathcal{C}}_{\infty, \mathfrak{f}_{\theta}}^{\chi} \otimes_{\mathcal{O}_{p}} M_{\theta \mathbb{Z}_{p}}(w+l)\right) \otimes_{\mathcal{O}_{p}[[\Gamma]]}^{\mathbb{L}} \mathcal{O}_{p}\right) \cong \operatorname{det}_{\mathcal{O}_{p}}\left(R \Gamma\left(\mathcal{O}_{S}, M_{\theta \mathbb{Z}_{p}}(w+l+1)\right)\right)^{-1}$.

Proof. In order to prove the theorem one can follow the same steps as in the proof of [22, Theorem 2.2.12], but with $M_{\theta \mathbb{Z}_{p}}(w)$ instead of $T_{p} E$. The only results that need some work are [22, Proposition 2.2.13] and [22, Lemma 2.2.16] (see $[2, \S 3.4]$ for a detailed proof). We will show next the necessary steps to prove these two results.

Let us prove the analog of [22, Proposition 2.2.13]:

$$
\operatorname{det}_{\mathcal{O}_{p}}\left(R \Gamma\left(\mathcal{G}, H^{0}\left(K_{\infty} \otimes \mathbb{Q}_{p}, M_{\theta \mathbb{Z}_{p}}(w+l+1)^{\prime}\right)\right)^{*}\right) \cong \mathcal{O}_{p}
$$

and

$$
\operatorname{det}_{\mathcal{O}_{p}}\left(R \Gamma\left(\mathcal{G}, H^{0}\left(\mathcal{O}_{\infty, S_{p}}, M_{\theta \mathbb{Z}_{p}}(w+l+1)^{\prime}\right)\right)^{*}\right) \cong \mathcal{O}_{p},
$$

where $M^{\prime}$ denotes $\operatorname{Hom}_{\mathcal{O}_{p}}\left(M, \mathcal{O}_{p} \otimes \mathbb{Q}_{p} / \mathbb{Z}_{p}(1)\right)$ and $M^{*}=H_{o m}{\mathcal{O}_{p}}_{p}\left(M, \mathcal{O}_{p} \otimes\right.$ $\left.\mathbb{Q}_{p} / \mathbb{Z}_{p}\right)$.

It follows from [12, prop. 2.4.6], that the action of $\mathcal{G}$ on $M_{\theta \mathbb{Z}_{p}}(w+l)$ is via the character

$$
\bar{\psi}_{\theta, p}: \mathcal{G} \rightarrow\left(\mathcal{O}_{\theta} \otimes \mathbb{Z}_{p}\right)^{*},
$$

multiplied by the $l$-th power of the $p$-adic cyclotomic character.

Then it induces a surjection of $\mathcal{O}_{p}$-modules $\rho: \mathcal{O}_{p}[[\Gamma]] \rightarrow M_{\theta \mathbb{Z}_{p}}(w+l)$ by the action described above. Thus $k \operatorname{er}(\rho)$ is an ideal of height 2 because $\Gamma \cong \mathbb{Z}_{p}^{2}$. We know that $\operatorname{det}_{R}$ is determined by the ideals of height 1 for the ring $R$ (cf. [19, 2.1.4]). We are going to show that this implies that

$$
\operatorname{det}_{\mathcal{O}_{p}}\left(M_{\theta \mathbb{Z}_{p}}(w+l) \otimes_{\mathcal{O}_{p}[[\mathcal{G}]]}^{\mathbb{L}} \mathcal{O}_{p}\right) \cong \mathcal{O}_{p}
$$

In fact, since $\Delta$ is finite and $\mathcal{G} \cong \Gamma \times \Delta$, we have the isomorphism

$$
M_{\theta \mathbb{Z}_{p}}(w+l) \otimes_{\mathcal{O}_{p}[[\mathcal{G}]]}^{\mathbb{L}} \mathcal{O}_{p} \cong\left(M_{\theta \mathbb{Z}_{p}}(w+l)\right)_{\Delta} \otimes_{\mathcal{O}_{p}[[\Gamma]]}^{\mathbb{L}} \mathcal{O}_{p}
$$

Since we know that $\operatorname{ker}(\rho)$ has height 2, we have that $\operatorname{det}_{\mathcal{O}_{p}[[\Gamma]]}\left(\left(M_{\theta \mathbb{Z}_{p}}(w+\right.\right.$ $\left.l))_{\Delta}\right) \cong \mathcal{O}_{p}[[\Gamma]]$ and so $\operatorname{det}_{\mathcal{O}_{p}}\left(\left(M_{\theta \mathbb{Z}_{p}}(w+l)\right)_{\Delta} \otimes_{\mathcal{O}_{p}[[\Gamma]]}^{\mathbb{L}} \mathcal{O}_{p}\right) \cong \mathcal{O}_{p}$. This shows (11). We conclude by using [22, Lemma 2.2.6]. 
Now, we show the analog of [22, Lemma 2.2.16]: the restriction map induces isomorphisms

$$
\begin{gathered}
\operatorname{det}_{\mathcal{O}_{p}}\left(H^{0}\left(\Delta, R \Gamma\left(\mathcal{O}_{0, S_{p}}, M_{\theta \mathbb{Z}_{p}}(w+l+1)\right)\right) \cong\right. \\
\operatorname{det}_{\mathcal{O}_{p}}\left(H^{0}\left(\Delta, R \Gamma\left(\mathcal{O}_{0, S}, M_{\theta \mathbb{Z}_{p}}(w+l+1)\right)\right)\right) \cong \operatorname{det}_{\mathcal{O}_{p}}\left(R \Gamma\left(\mathcal{O}_{S}, M_{\theta \mathbb{Z}_{p}}(w+l+1)\right)\right) .
\end{gathered}
$$

To show this consider the exact triangle

$$
\begin{gathered}
R \Gamma\left(\mathcal{O}_{0, S_{p}}, M_{\theta \mathbb{Z}_{p}}(w+l+1)\right) \rightarrow R \Gamma\left(\mathcal{O}_{0, S}, M_{\theta \mathbb{Z}_{p}}(w+l+1)\right) \\
\rightarrow \oplus_{v_{0} \in S \backslash S_{p}} R \Gamma_{k\left(v_{0}\right)}\left(\mathcal{O}_{v}, M_{\theta \mathbb{Z}_{p}}(w+l)\right)[1]
\end{gathered}
$$

where $\mathcal{O}_{v_{0}}$ is the local ring at $v_{0}$ and $S_{p}$ is the set of places that divide $p$. Since $T_{p} E$ is unramified at the places of $K_{0}$ in $S \backslash S_{p}$, the same is true for $e_{\theta}\left(\otimes T_{p} E\right)(l+1)$. By the purity theorems in étale cohomology we have that

$$
R \Gamma_{k\left(v_{0}\right)}\left(\mathcal{O}_{v_{0}}, M_{\theta \mathbb{Z}_{p}}(w+l+1)\right) \cong R \Gamma\left(k\left(v_{0}\right), M_{\theta \mathbb{Z}_{p}}(w+l)\right)[-2] .
$$

It remains to prove only that

$$
H^{0}\left(\Delta, \oplus_{v_{0} \in S \backslash S_{p}} R \Gamma\left(k\left(v_{0}\right), M_{\theta \mathbb{Z}_{p}}(w+l)\right)=0 .\right.
$$

To show this result, observe that

$$
H^{1}\left(k\left(v_{0}\right), M_{\theta \mathbb{Z}_{p}}(w+l)\right) \cong M_{\theta \mathbb{Z}_{p}}(w+l)_{G a l\left(\overline{k\left(v_{0}\right)} / k\left(v_{0}\right)\right)}
$$

and $H^{0}\left(k\left(v_{0}\right), M_{\theta \mathbb{Z}_{p}}(w+l)\right)=0$ because $-w-2 l \leq-3$.

Now, let $v_{0}$ be a prime of $K_{0}$ dividing $v$ a prime of $K$ with $v \mid \mathfrak{f}_{\theta}$ and let $\Delta_{v_{0}}$ be the stabilizer of $v_{0}$. Since $I_{v_{0}} \subset \Delta_{v_{0}}$ acts non trivially on the coinvariants $M_{\theta \mathbb{Z}_{p}}(w+l)_{\operatorname{Gal}\left(\overline{k\left(v_{0}\right)} / k\left(v_{0}\right)\right)}$ because $v_{0} \mid \mathfrak{f}_{\theta}$, there are no fix elements.

\section{The comparison between the map $r_{p}$ and $(\text { Soul })_{p}$ in the constructible $K$-elements}

Let's start recalling the result of Kings on the specialization of the elliptic polylogarithm sheaf, which is an important key in his proof of the Tamagawa number conjecture.

Let $E$ be an elliptic curve over a base scheme $T$, and denote by $\bar{\pi}: E \rightarrow T$ the structural morphism, which is proper and smooth. Consider $U=E \backslash e$, where $e$ is the zero section of $E$. Consider the elliptic polylogarithm sheaf $\mathcal{P}$ ol $\mathbb{Q}_{p}$ on $U$, which is a lisse pro-sheaf (i.e. a projective limits of lisse sheaves) [22, §3.2].

Let $\mathcal{H}_{\mathbb{Q}_{p}}:=\underline{\operatorname{Hom}}_{T}\left(R^{1} \bar{\pi}_{*} \mathbb{Q}_{p}, \mathbb{Q}_{p}\right)$. Using $\mathcal{P}$ ol $\mathbb{Q}_{p}$ one defines the $p$-adic Eisenstein classes associated to any integer $k$ and any $M$-torsion point $t$ in $E$ different from $e$ as some elements in $H^{1}\left(T, S m^{k} \mathcal{H}_{\mathbb{Q}_{p}}\right)$. The definition is extended by linearity to any divisor supported on $M$-torsion points ([22, Def. 3.5.9]). The main part of the result of Kings is the explicit computation of these Eisenstein classes.

We are going to explain this result. Consider $H_{n}:=\operatorname{ker}\left[p^{n}\right]$ as a scheme over $T$. Let us consider the map multiplication by $p^{n}, p_{n}: E_{n} \rightarrow E$, where $E_{n}$ is the elliptic curve $E$ over $T$ considered as a $H_{n}$-torsor over $E$. Consider the characteristic group $I\left[H_{n}\right]:=\operatorname{ker}\left(p_{n, *} \mathbb{Z} \rightarrow \mathbb{Z}\right)$, which is the characteristic 
group of a torus $T_{H_{n}}$. In this situation we have the connecting map $\delta$ from the Kummer exact sequence:

$$
\delta: H^{0}\left(H_{n}, T_{H_{n}}\right) \rightarrow H^{1}\left(H_{n}, T_{H_{n}}\left[p^{r}\right]\right) .
$$

Using this connecting morphism, we can express the Eisenstein classes explicitly.

Theorem 5.1 (Kings, theorem 4.2.9 in [22]). Let $p$ be a prime number, and let $E$ be an elliptic curve over a base scheme $T$ where $p$ is invertible.

Let $\beta$ be any divisor in $E$ of the form

$$
\beta:=\sum_{t \in E[M](T) \backslash e} n_{t}(t),
$$

$n_{t}$ an integer and consider $[\mathfrak{a}]: E \rightarrow E$ any isogeny with degree prime to Mp.

Then, for any $m>0$, the $p$-adic Eisenstein class

$$
N \mathfrak{a}\left(\mathfrak{a}^{\otimes m} N \mathfrak{a}-1\right)\left(\beta^{*} \operatorname{Pol}_{\mathbb{Q}_{p}}\right)^{m} \in H^{1}\left(T, \operatorname{Sym}^{m} \mathcal{H}_{\mathbb{Q}_{p}}(1)\right)
$$

is given by

$$
\pm \frac{1}{m !}\left(\delta \sum_{t \in E[M](T) \backslash e} n_{t} \sum_{\left[p^{n}\right] t_{n}=t} \theta_{\mathfrak{a}}\left(-t_{n}\right) \widetilde{t_{n}}{ }^{\otimes m}\right)_{n}
$$

where $\widetilde{t_{n}}$ is the projection of $t_{n}$ to $E\left[p^{n}\right]$ and $\delta$ is the Sym-extension of the boundary map $H^{0}\left(H_{n}, T_{H_{n}}\right) \rightarrow H^{1}\left(H_{n}, T_{H_{n}}\left[p^{r}\right]\right)$ where $H_{n}:=k e r\left[p^{n}\right]$ is considered as a scheme over $T$ and $T_{H_{n}}$ is the torus with character group $I\left[H_{n}\right]:=$ $\operatorname{ker}\left(p_{n, *} \mathbb{Z} \rightarrow \mathbb{Z}\right)$.

The following result relates the image of $\mathcal{E}_{\mathcal{M}}^{m}(\beta)$ by the Soulé regulator map with the polylogarithmic sheaf, where $\mathcal{E}_{\mathcal{M}}^{m}$ is the Eisenstein symbol [22, §1.2.1].

Theorem 5.2. Under the same hypothesis of Theorem 5.1, let $\beta$ be as in the previous theorem. Then

$$
r_{p}\left(\mathcal{E}_{\mathcal{M}}^{m}(\beta)\right)=-M^{2 m}\left(\beta^{*} \text { Pol }_{\mathbb{Q}_{p}}\right)^{m}
$$

in $H^{1}\left(T, \operatorname{Sym}^{m} \mathcal{H}_{\mathbb{Q}_{p}}(1)\right)$.

Proof. The same proof of [22, Theorem 1.2.5] with $m$ instead of $2 k+1$ works. See also [2, proof Theorem 3.5.2].

We are going to apply these results to the divisor $\beta_{\theta}=N_{K(\mathfrak{f}) / K}((t))$, where $t:=\Omega f^{-1}$ is a $\mathfrak{f}_{\theta}$-torsion point. Take $M=N_{K / \mathbb{Q}} \mathfrak{f}_{\theta}, m=w+2 l, T=\mathcal{O}_{S}$ and $\mathcal{H}_{\mathbb{Q}_{p}}=T_{p} E \otimes \mathbb{Q}_{p}$, using the notations of the theorem 5.1. Let $\mathfrak{a} \subset \mathcal{O}_{K}$ be an ideal prime to $6 p \mathfrak{f}$, and consider the isogeny given by $\varphi(\mathfrak{a})$. Let $\theta_{\mathfrak{a}}$ be the classical theta function.

To simplify the notation, define for any $\widetilde{t_{r}} \in E\left[p^{r}\right]$

$$
\gamma\left(\widetilde{t_{r}}\right)^{m}:=<\widetilde{t_{r}}, \sqrt{d_{K}} \widetilde{t_{r}}>^{\otimes m}
$$

where $<,>$ denotes the Weil pairing. Our objective is the computation of

$$
\mathcal{K}_{\mathcal{M}} \circ \mathcal{E}_{\mathcal{M}}^{w+2 l}\left(\beta_{\theta}\right) .
$$

Remember that we are under the restriction $a_{\theta} \not \equiv b_{\theta} \bmod \left(\# \mathcal{O}_{K}^{*}\right)$.

We consider the following commutative diagram $[8,(2.8)]$ 


$$
\begin{array}{lcr}
H_{\mathcal{M}}^{2 l+w+1}\left(S_{y m^{2 l+w}} h^{1} E, \mathbb{Q}(w+2 l+1)\right) & \left(\left(\Delta_{C M}\right)^{l} \times i d\right)^{*} & H_{\mathcal{M}}^{2 l+w+1}\left(E^{l+w}, \mathbb{Q}(2 l+w+1)\right) \\
\mathcal{K}_{\mathcal{M}} \downarrow & \stackrel{e_{\theta}}{\longleftarrow} & H_{\mathcal{M}}^{w+1}\left(h^{1}(E)^{\otimes w}, \mathbb{Q}(l+w+1)\right), \\
H_{\mathcal{M}}^{w+1}\left(M_{\theta \mathbb{Q}}, \mathbb{Q}(w+l+1)\right) & &
\end{array}
$$

where $p r$ is the projection in the last $w$ components and $\Delta_{C M}: E \rightarrow E \times E$ is given by $e \mapsto\left(e, \sqrt{d_{K}} e\right)$. We obtain a map in Galois cohomology given by

$$
\begin{gathered}
H^{1}\left(\mathcal{O}_{S}, \operatorname{Sym}^{2 l+w} \mathcal{H}_{\mathbb{Q}_{p}}(1)\right) \rightarrow \\
H^{1}\left(\mathcal{O}_{S},\left(e_{\theta} \mathcal{S}_{y m^{w}} \mathcal{H}_{\mathbb{Q}_{p}}\right)(l+1)\right)=H^{1}\left(\mathcal{O}_{S}, M_{\theta \mathbb{Q}_{p}}(w+l+1)\right)
\end{gathered}
$$

such that

$$
\mathcal{K}_{\mathcal{M}}\left(\varphi_{p}(\mathfrak{a})^{\otimes 2 l+w} \operatorname{Sym}^{2 l+w} \mathcal{H}_{\mathbb{Q}_{p}}(1)\right)=e_{\theta}\left(\otimes^{w} \varphi_{p}(\mathfrak{a})\right) N \mathfrak{a}^{l} \operatorname{Sym}^{w} \mathcal{H}_{\mathbb{Q}_{p}}(l+1) .
$$

Theorem 5.3. Let $p$ be a prime number such that $p \nmid 6 N_{K / \mathbb{Q}}(\mathfrak{f})$. Let $\theta$ be an idempotent with infinity type $\left(a_{\theta}, b_{\theta}\right)$ which $a_{\theta} \not \equiv b_{\theta} \bmod \left(\# \mathcal{O}_{K}^{*}\right)$. For a $p^{r} N_{K / \mathbb{Q}}\left(\mathfrak{f}_{\theta}\right)$-torsion point $t_{r}$, denote by $\widetilde{t_{r}}$ its projection to $E\left[p^{r}\right]$. Then, if $t=$ $\Omega f^{-1}$, we have the following equality

$$
\begin{gathered}
N \mathfrak{a}\left(\psi_{\theta, p}(\mathfrak{a}) N \mathfrak{a}^{l+1}-1\right) r_{p}\left(\xi_{\theta, l}\right)= \\
\frac{(-1)^{l} L_{p}\left(\bar{\psi}_{\theta},-l\right)^{-1} N_{T_{\theta} / \mathbb{Q}} \mathfrak{f}_{\theta}^{3 l+2 w} \Phi\left(\mathfrak{f}_{\theta}\right)}{2^{l-1} \psi_{\theta}\left(\rho_{\theta}\right) \Phi(\mathfrak{f})} \cdot\left(\left(\delta N_{K(\mathfrak{f}) / K} \sum_{p^{r} t_{r}=t} \theta_{\mathfrak{a}}\left(-t_{r}\right) \otimes e_{\theta}\left(\otimes^{w} \widetilde{t_{r}}\right) \otimes \gamma\left(\widetilde{t_{r}}\right)^{l}\right)_{r}\right.
\end{gathered}
$$

Proof. Using theorems 3.5, 5.1 and 5.2, we have that

$$
\begin{gathered}
r_{p}\left(\xi_{\theta, l}\right)=\frac{(-1)^{l-1}(2 l+w) ! L_{p}\left(\bar{\psi}_{\theta},-l\right)^{-1} \Phi\left(\mathfrak{f}_{\theta}\right)}{2^{l-1} N_{T_{\theta} / \mathbb{Q}} \mathfrak{f}_{\theta}^{l} \psi_{\theta}\left(\rho_{\theta}\right) \Phi(\mathfrak{f})} \mathcal{K}_{\mathcal{M}}\left(\mathcal{E}_{\mathcal{M}}^{2 l+w}(\beta)\right) \\
\left.=\frac{(-1)^{l}(2 l+w) ! L_{p}\left(\bar{\psi}_{\theta},-l\right)^{-1} N_{T_{\theta} / \mathbb{Q} f_{\theta}^{3 l+2 w} \Phi\left(\mathfrak{f}_{\theta}\right)} \mathcal{K}_{\mathcal{M}}\left(\beta^{*} P^{l-1} \psi_{\theta}\left(\rho_{\theta}\right) \Phi\left(\mathbb{Q}_{p}\right)\right.}{}\right)^{w+2 l} .
\end{gathered}
$$

We have that

$$
\mathcal{K}_{\mathcal{M}}\left(\widetilde{t_{r}}{ }^{\otimes 2 l+w}\right)=e_{\theta}\left(\otimes^{w} \widetilde{t_{r}}\right) \otimes \gamma\left(\widetilde{t_{r}}\right)^{l} .
$$

Finally, applying Kings' theorem [5.1, we obtain the desired identity.

We want to rewrite the previous formula in terms of the norm map of the extension $K\left(\mathfrak{f}_{\theta}\right) K\left(E\left[p^{n}\right]\right) / K$. We will work with $\mathfrak{f}$ instead of $\mathfrak{f}_{\theta}$ since then we can use that $K\left(E\left[\mathfrak{p}^{n} \mathfrak{f}\right]\right)=K\left(\mathfrak{p}^{\mathfrak{n}} \mathfrak{f}\right)$, the ray class field, because $\mathfrak{f}$ is the conductor of $E$ and divides the ideal $\mathfrak{f p}^{n}$ ([9, II, Prop.1.6]). by

Fix a prime $\mathfrak{p}$ of $K$ where $E$ has good reduction, and take $\pi=\varphi(\mathfrak{p})$. Denote

$$
H_{r, t}^{\mathfrak{p}}:=\left\{t_{r} \in E\left[\mathfrak{p}^{r} \mathfrak{f}\right] \mid \pi^{r} t_{r}=t\right\} .
$$

Let $\sigma_{\mathfrak{p}}$ be the Frobenius at $\mathfrak{p}$ in $\operatorname{Gal}(K(\mathfrak{f}) / K)$, we write $t_{r}=\left(\widetilde{t_{r}}, \pi^{-r} t\right) \in E\left[\mathfrak{p}^{r} \mathfrak{f}\right]=$ $E\left[\mathfrak{p}^{r}\right] \oplus E[\mathfrak{f}]$, where $\pi^{-r} t$ means $t^{\sigma_{\mathfrak{p}}^{-r}}$.

Consider the filtration of $H_{r, s}^{\mathfrak{p}}$ defined by

$$
F_{r, t}^{i}:=\left\{t_{r} \in H_{r, s}^{\mathfrak{p}} \mid \pi^{r-i} \widetilde{t_{r}}=0\right\} .
$$


Theorem 5.4. Let $\mathfrak{p}$ be as above and $t_{r}=\left(\widetilde{t_{r}}, \pi^{-r} t\right) \in F_{r, s}^{0} \backslash F_{s, t}^{1}$. Suppose that $\mathcal{O}_{K}^{*} \rightarrow\left(\mathcal{O}_{K} / \mathfrak{f}_{\theta}\right)^{*}$ is injective. Denote the Euler factor of the Hecke character $\bar{\psi}_{\theta}$ at $\mathfrak{p}$ evaluated at $-l$ by $L_{\mathfrak{p}}\left(\overline{\psi_{\theta}},-l\right)$. Then

$$
\begin{gathered}
L_{\mathfrak{p}}\left(\bar{\psi}_{\theta},-l\right)^{-1}\left(N_{K(\mathfrak{f}) / K} \sum_{s_{r} \in H_{r, t}^{\mathfrak{p}}} \theta_{\mathfrak{a}}\left(-s_{r}\right) \otimes e_{\theta}\left(\otimes^{w} \widetilde{s_{r}}\right) \otimes \gamma\left(\widetilde{s_{r}}\right)^{l}\right)_{r}= \\
\left(N_{K\left(\mathfrak{p}^{r} \mathfrak{f}\right) / K}\left(\theta_{\mathfrak{a}}\left(-t_{r}\right) \otimes e_{\theta}\left(\otimes^{w} \widetilde{t_{r}}\right) \otimes \gamma\left(\widetilde{t_{r}}\right)^{l}\right)\right)_{r}
\end{gathered}
$$

in $H^{1}\left(\mathcal{O}_{S}, e_{\theta}\left(T_{\mathfrak{p}} E(1)\right)(l) \otimes \mathbb{Q}_{p}\right)$ for all $\mathfrak{a}$ relatively prime to $\mathfrak{p} \mathfrak{f}$.

Proof. The identification $\operatorname{Hom}_{\mathcal{O}_{p}}\left(T_{p} E, \mathcal{O}_{p}\right) \cong T_{p} E(-1)$ is via the conjugate linear $\mathcal{O}_{p}$-action on the right side. Hence $\overline{\varphi(\mathfrak{p})} t_{r}=t_{r-1}$. We have the equality

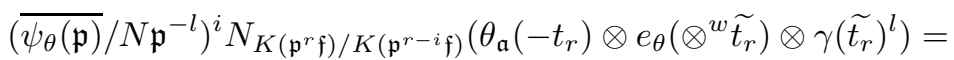

$$
\begin{aligned}
& N_{K\left(\mathfrak{p}^{r} \mathfrak{f}\right) / K\left(\mathfrak{p}^{r-i} \mathfrak{f}\right)}\left(\theta_{\mathfrak{a}}\left(-t_{r}\right) \otimes e_{\theta}\left(\otimes^{w} \overline{\varphi(\mathfrak{p})}^{i} \tilde{t_{r}}\right) \otimes \gamma\left(\overline{\varphi(\mathfrak{p})} \tilde{t}^{i}\right)^{l}\right)= \\
& \left.\left(N_{K\left(\mathfrak{p}^{r} \mathfrak{f}\right) / K\left(\mathfrak{p}^{r-i} \mathfrak{f}\right)}\left(\theta_{\mathfrak{a}}\left(-t_{r}\right)\right)\right) \otimes e_{\theta}\left(\otimes^{w} \widetilde{t_{r-i}}\right) \otimes \gamma\left(\widetilde{t_{r-1}}\right)^{l}\right)= \\
& \theta_{\mathfrak{a}}\left(-\left(\widetilde{t_{r-i}}, \pi^{i-r} t\right)\right) \otimes e_{\theta}\left(\otimes^{w} \widetilde{t_{r-i}}\right) \otimes \gamma\left(\widetilde{t_{r-i}}\right)^{l},
\end{aligned}
$$

where the last equality uses the distribution relation for $\theta_{\mathfrak{a}}$ ([9, II 2.5]).

The Galois group of $K\left(\mathfrak{p}^{r-i} \mathfrak{f}\right) / K(\mathfrak{f})$ acts simply transitively on $F_{r, t}^{i} \backslash F_{r, t}^{i+1}$. We get that

$$
\begin{gathered}
\left(\overline{\psi_{\theta}(\mathfrak{p})} / N \mathfrak{p}^{-l}\right)^{i} N_{K\left(\mathfrak{p}^{r} \mathfrak{f}\right) / K(\mathfrak{f})}\left(\theta_{\mathfrak{a}}\left(-t_{r}\right) \otimes e_{\theta}\left(\otimes^{w} \widetilde{t_{r}}\right) \otimes \gamma\left(\widetilde{t_{r}}\right)^{l}\right)= \\
\sum_{t_{r-i} \in F_{r, t}^{i} \backslash F_{r, t}^{i+1}} \theta_{\mathfrak{a}}\left(-\left(\widetilde{t_{r-i}}, \pi^{i-r} t\right)\right) \otimes e_{\theta}\left(\otimes \widetilde{t_{r-i}}\right) \otimes \gamma\left(\widetilde{t_{r-i}}\right)^{l} .
\end{gathered}
$$

We know by [9, Prop. II.2.4.ii)] that we have the equality $\theta_{\mathfrak{a}}\left(-\left(\widetilde{t_{r-i}}, \pi^{i-r} t\right)\right)=$ $\theta_{\mathfrak{a}}\left(-\left(\widetilde{t_{r-i}}, \pi^{-r} t\right)\right)^{\sigma_{\mathfrak{p}}^{i}}$ with $\sigma_{\mathfrak{p}}$ is the Frobenius at $\mathfrak{p}$ in the Galois group of $K(\mathfrak{f}) / K$. This and the fact that $N_{K(\mathfrak{f}) / K}$ is the sum over all Galois translates, which act trivially on $\widetilde{t_{r-i}}$, gives that

$$
\begin{gathered}
\left(\overline{\psi_{\theta}(\mathfrak{p})} / N \mathfrak{p}^{-l}\right)^{i} N_{K\left(\mathfrak{p}^{r} \mathfrak{f}\right) / K}\left(\theta_{\mathfrak{a}}\left(-t_{r}\right) \otimes e_{\theta}\left(\otimes^{w} \widetilde{t_{r}}\right) \otimes \gamma\left(\widetilde{t_{r}}\right)^{l}\right)= \\
\left.N_{K(\mathfrak{f}) / K}\left(\sum_{t_{r-i} \in F_{r, t}^{i} \backslash F_{r, t}^{i+1}} \theta_{\mathfrak{a}}\left(-\widetilde{\left(\widetilde{t_{r-i}}\right.}, \pi^{-r} t\right)\right) \otimes e_{\theta}\left(\otimes^{w} \widetilde{t_{r-i}}\right) \otimes \gamma\left(\widetilde{t_{r-i}}\right)^{l}\right),
\end{gathered}
$$

Adding these equalities with respect to $i$ and increasing $r$ if necessary we get the result.

Lemma 5.5. Suppose that $\theta$ has infinity type $(w, 0)$ or $(0, w)$ and $\left(\# \mathcal{O}_{K}^{*}, w\right)=$ 1. Then

$$
\mathcal{O}_{K}^{*} \rightarrow\left(\mathcal{O}_{K} / \mathfrak{f}_{\theta}\right)^{*}
$$

is injective. 
Proof. Let $u$ be and element in $\mathcal{O}_{K}^{*}, u \neq 1$ and consider the idèle defined by $x_{\infty}=1$ and $x_{\mathfrak{p}}=u$ at all finite places $\mathfrak{p}$ of $K$. Then as complex Hecke character $\varphi^{w}(x)=\varphi^{w}\left(u^{-1} x\right)=u^{w} \neq 1$ if $\left(w, \# \mathcal{O}_{K}^{*}\right)=1$. So, by definition of the conductor of $\psi_{\theta}$, we obtain that $u \neq \equiv 1\left(\bmod \mathfrak{f}_{\theta}\right)$, hence the result for the type $(w, 0)$. For the type $(0, w)$ the proof is similar but with $\bar{\varphi}$ instead of $\varphi$.

Corollary 5.6. Suppose $p \nmid 6 N_{K / \mathbb{Q}}(\mathfrak{f}), \mathcal{O}_{K}^{*} \rightarrow\left(\mathcal{O}_{K} / \mathfrak{f}_{\theta}\right)^{*}$ is injective and the hypothesis of theorem 5.3. Then

$$
\begin{gathered}
N \mathfrak{a}\left(\psi_{\theta, p}(\mathfrak{a}) N \mathfrak{a}^{l+1}-1\right) r_{p}\left(\xi_{\theta, l}\right)= \\
\pm \frac{N_{K / \mathbb{Q}}\left(\mathfrak{f}_{\theta}\right)^{3 l+2 w} \Phi\left(\mathfrak{f}_{\theta}\right)}{2^{l-1} \psi_{\theta}\left(\rho_{\theta}\right) \Phi(\mathfrak{f})} \delta\left(N_{K\left(E\left[p^{r}\right]\right) K(\mathfrak{f}) / K} \theta_{\mathfrak{a}}\left(-t_{r}\right) \otimes e_{\theta}\left(\otimes^{w} \widetilde{t_{r}}\right) \otimes \gamma\left(\widetilde{t_{r}}\right)^{l}\right)_{r}= \\
\pm \frac{N_{K \mathbb{Q}}\left(\mathfrak{f}_{\theta}\right)^{3 l+2 w}}{2^{l-1} \psi_{\theta}\left(\rho_{\theta}\right)} \cdot \delta\left(N_{K\left(E\left[p^{r}\right]\right) K\left(\mathfrak{f}_{\theta}\right) / K} \theta_{\mathfrak{a}}\left(-t_{r}\right) \otimes e_{\theta}\left(\otimes{ }^{w} \widetilde{t_{r}}\right) \otimes \gamma\left(\widetilde{t_{r}}\right)^{l}\right)_{r}
\end{gathered}
$$

where $t_{r}$ is a primitive $p^{r} \mathfrak{f}_{\theta}$-torsion point with $p^{r} t_{r}=t$ and $\mathfrak{a}$ is relative prime to $p \mathfrak{f}$.

Proof. If $p$ is inert or prime the first equality is deduced from the previous theorem. If $p$ split, it decomposes in a $\mathfrak{p}$ part and a $\mathfrak{p}^{*}$ part. Putting together the previous result with $\mathfrak{p}$ and with $\mathfrak{p}^{*}$, we have the first equality.

To show the second equality, consider

$$
\begin{gathered}
N_{K\left(E\left[p^{r}\right]\right) K(\mathfrak{f}) / K\left(E\left[p^{r}\right]\right) K\left(\mathfrak{f}_{\theta}\right)} \theta_{\mathfrak{a}}\left(-t_{r}\right)= \\
\prod_{\sigma \in \operatorname{Gal}\left(K(\mathfrak{f}) K\left(E\left[p^{r}\right]\right) K\left(p^{r}\right) / K\left(\mathfrak{f}_{\theta}\right) K\left(E\left[p^{r}\right]\right) K\left(p^{r}\right)\right)} \theta_{\mathfrak{a}}\left(-t_{r}\right)^{\sigma}
\end{gathered}
$$

because $K(\mathfrak{f})$ is disjoint with $K\left(p^{r}\right)$ over $K$ since $K=K(1)$, and $K(\mathfrak{f})=K(E[\mathfrak{f}])$ is disjoint with $K\left(E\left[p^{r}\right]\right)$ over $K$. Moreover, since $\theta_{\mathfrak{a}}\left(-t_{r}\right) \in K(\mathfrak{f}) K\left(p^{r}\right)=$ $K\left(\mathfrak{f} p^{r}\right)$ and $(\mathfrak{f}, p)=1$, we have that the norm is equal to

$$
\prod_{\tau \in \operatorname{Gal}\left(K\left(\mathfrak{f} p^{r}\right) / K\left(\mathfrak{f}_{\theta} p^{r}\right)\right)} \theta_{\mathfrak{a}}\left(-t_{r}\right)^{\tau} .
$$

But $\theta_{\mathfrak{a}}\left(-t_{r}\right) \in K\left(\mathfrak{f}_{\theta} p^{r}\right)$ because $-t_{r}$ is a point of $\mathfrak{f}_{\theta} p^{r}$-torsion. To obtain the second equality we need to show that $\frac{\Phi\left(\mathfrak{f}_{\theta}\right)}{\Phi(\mathfrak{f})} \#\left(\operatorname{Gal}\left(K(\mathfrak{f}) / K\left(\mathfrak{f}_{\theta}\right)\right)\right)$ is one. We have $\frac{\Phi\left(\mathfrak{f}_{\theta}\right)}{\Phi(\mathfrak{f})} \#\left(G a l\left(K(\mathfrak{f}) / K\left(\mathfrak{f}_{\theta}\right)\right)\right)=\frac{\Phi\left(\mathfrak{f}_{\theta}\right)}{\Phi(\mathfrak{f})} \frac{[K(\mathfrak{f}): K(1)]}{\left[K\left(f_{\theta}\right): K(1)\right]}$, we are in Galois extensions. Observe that $\frac{\Phi\left(\mathfrak{f}_{\theta}\right)}{\Phi(\mathfrak{f})} \frac{[K(\mathfrak{f}): K(1)]}{\left[K\left(f_{\theta}\right): K(1)\right]}=\frac{\Phi\left(\mathfrak{f}_{\theta}\right)}{\Phi(\mathfrak{f})} \frac{\Phi(\mathfrak{f}) \frac{w_{\mathfrak{f}}}{w_{K}}}{\Phi\left(\mathfrak{f}_{\theta}\right) \frac{w_{\mathfrak{f}_{\theta}}}{w_{K}}}$ where $w_{K}$ are the roots of unity of $K$ and $w_{\mathfrak{m}}$ are the roots of unit of $\mathcal{O}_{K}^{*}$ congruent to 1 modulo $\mathfrak{m}$, by class field theory (see [9, p.36]). And is equal to one because $\mathcal{O}^{*} \rightarrow\left(\mathcal{O}_{K} / \mathfrak{m}\right)^{*}$ is injective for $\mathfrak{m}=\mathfrak{f}_{\theta}$ by hypothesis, and for $\mathfrak{m}=\mathfrak{f}($ Lemma [5.5] or [25. Corollary 5.9]).

Now we want to show that the elements

$$
\left(N_{K\left(E\left[p^{r}\right]\right) K\left(\mathfrak{f}_{\theta}\right) / K} \theta_{\mathfrak{a}}\left(t_{r}\right) \otimes e_{\theta}\left(\otimes^{w} \widetilde{t_{r}}\right) \otimes \gamma\left(\widetilde{t_{r}}\right)^{l}\right)_{r}
$$

generate $\left(\overline{\mathcal{C}}_{\infty}^{\chi} \otimes M_{\theta \mathbb{Z}_{p}}(w+l)\right)_{\Gamma}$, where $\mathfrak{a}$ is prime to $6 p \mathfrak{f}$ and $\chi$ is the representation of $\Delta$ on $\operatorname{Hom}_{\mathcal{O}_{p}}\left(M_{\theta \mathbb{Z}_{p}}(w+l), \mathcal{O}_{p}\right)$, that we suppose a good representation.

We suppose from now on that the natural map $\mathcal{O}_{K}^{*} \rightarrow\left(\mathcal{O}_{K} / \mathfrak{f}_{\theta}\right)^{*}$ is injective, assumption also needed to define our elliptic units. 
Proposition 5.7. Consider $p \nmid 6 N_{K / \mathbb{Q}}(\mathfrak{f})$ and $\mathfrak{a}$ an ideal in $\mathcal{O}_{p}$, which is prime to $6 p \mathfrak{f}$ and such that $\psi_{\theta, p}(\mathfrak{a}) N \mathfrak{a}^{l+1} \not \equiv 1(\bmod p)$. Then the $\mathcal{O}_{p}[[\Gamma]]-$ module

$$
\overline{\mathcal{C}}_{\infty, \mathfrak{f}_{\theta}}^{\chi} \otimes \mathcal{O}_{p} M_{\theta \mathbb{Z}_{p}}(w+l)
$$

is generated by $\left(\theta_{\mathfrak{a}}\left(t_{r}\right) \otimes e_{\theta}\left(\otimes^{w} \widetilde{t_{r}}\right) \otimes \gamma\left(\widetilde{t_{r}}\right)^{l}\right)_{r}$, where $t_{r}$ is a primitive $p^{r} \mathfrak{f}_{\theta}$-division point.

Remark 5.8. The existence of an ideal $\mathfrak{a}$ satisfying the conditions of the proposition 5.7 is equivalent to the condition that the $\Delta$-representation $\chi$ is not the cyclotomic representation.

Proof. Observe first that $e_{\theta}\left(\widetilde{t_{r}}\right) \otimes \gamma\left(\widetilde{t_{r}}\right)$ generates $M_{\theta \mathbb{Z}_{p}}(w+l)$, because $M_{\theta \mathbb{Z}_{p}}(w)$ is one dimensional and concerning how it generates $\mathbb{Z}_{p}(l)$ use the same proof did in [22, p.623].

Remember that we have an inclusion of $\overline{\mathcal{C}}_{\infty, \mathfrak{f}_{\theta}}^{\chi}$ in $\mathcal{U}_{\infty}$ the local units Iwasawa module, which is torsion free [25, Prop.11.4], thus $\overline{\mathcal{C}}_{\infty, \mathfrak{f}_{\theta}}^{\chi}$ is a torsion free $\mathcal{O}_{p}[[\Gamma]]$ module. Is enough to show that is one dimensional. Let $\mathfrak{b}$ be another ideal prime to $6 p \mathfrak{f}$. Take $\sigma_{\mathfrak{a}}=\left[\mathfrak{a}, K_{n} / K\right]$ and $\sigma_{\mathfrak{b}}=\left[\mathfrak{b}, K_{n} / K\right]$. Then, by the properties of the theta function, we have that

$$
\begin{gathered}
\left(\sigma_{\mathfrak{a}}-\psi_{\theta, p}(\mathfrak{a}) N \mathfrak{a}^{l+1}\right)\left(\theta_{\mathfrak{b}}\left(t_{n}\right) \otimes e_{\theta}\left(\otimes^{w} \widetilde{t_{n}}\right) \otimes \gamma\left(\widetilde{t_{n}}\right)^{l}\right)= \\
\psi_{\theta, p}(\mathfrak{a}) N \mathfrak{a}^{l}\left(\theta_{\mathfrak{b}}\left(t_{n}\right)^{\sigma_{\mathfrak{a}}-N \mathfrak{a}} \otimes e_{\theta}\left(\otimes^{w} \widetilde{t_{n}}\right) \otimes \gamma\left(\widetilde{t_{n}}\right)^{l}\right)= \\
\psi_{\theta, p}(\mathfrak{a}) N \mathfrak{a}^{l}\left(\theta_{\mathfrak{a}}\left(t_{n}\right)^{\sigma_{\mathfrak{b}}-N \mathfrak{b}} \otimes e_{\theta}\left(\otimes^{w} \widetilde{t_{n}}\right) \otimes \gamma\left(\widetilde{t_{n}}\right)^{l}\right) .
\end{gathered}
$$

Then, it is enough show that $\left(\sigma_{\mathfrak{a}}-\psi_{\theta, p}(\mathfrak{a}) N \mathfrak{a}^{l+1}\right)$ is invertible in $\mathcal{O}_{p}[[\Gamma]]$. But the element $\sigma_{\mathfrak{a}}$ corresponds to 1 on $\mathcal{O}_{p} / p$ and thus $\sigma_{\mathfrak{a}}-\psi_{\theta}(\mathfrak{a}) N \mathfrak{a}^{l+1}$ is invertible in $\mathcal{O}_{p}[[\Gamma]]$ because $1 \not \equiv \psi_{\theta}(\mathfrak{a}) N \mathfrak{a}^{l+1} \bmod p$.

Corollary 5.9. Assume that $p \nmid 6 N_{K / \mathbb{Q}} \mathfrak{f}$. Then the image of $\mathcal{R}_{\theta}$ by $r_{p}$ in the cohomology group $H^{1}\left(\mathcal{O}_{S}, M_{\theta \mathbb{Z}_{p}}(w+l+1)\right) \otimes \mathbb{Q}_{p}$ coincides with

$$
(\operatorname{Soul})_{p}\left(\left(\overline{\mathcal{C}}_{\infty, \mathfrak{f}_{\theta}}^{\chi} \otimes M_{\theta \mathbb{Z}_{p}}(w+l)\right)_{\Gamma}\right) .
$$

Proof. As

$$
N \mathfrak{f}_{\theta}^{3 l+2 w} / 2^{l-1} \psi_{\theta}\left(\rho_{\theta}\right)
$$

is prime to $p$, it follows from the definition of $(\text { Soul })_{p}$ and Corollary 5.6.

Lemma 5.10. The canonical map

$\left(\overline{\mathcal{C}}_{\infty, \mathfrak{f}_{\theta}} \otimes M_{\theta \mathbb{Z}_{p}}(w+l)\right) \otimes_{\mathcal{O}_{p}[[\mathcal{G}]]}^{\mathbb{L}} \mathcal{O}_{p} \rightarrow\left(\overline{\mathcal{C}}_{\infty, \mathfrak{f}_{\theta}} \otimes M_{\theta \mathbb{Z}_{p}}(w+l)\right)_{\mathcal{G}} \cong\left(\overline{\mathcal{C}}_{\infty, \mathfrak{f}_{\theta}}^{\chi} \otimes M_{\theta \mathbb{Z}_{p}}(w+l)\right)_{\Gamma}$

is an isomorphism and moreover $\left(\overline{\mathcal{C}}_{\infty, \mathfrak{f}_{\theta}}^{\chi} \otimes M_{\theta \mathbb{Z}_{p}}(w+l)\right)_{\Gamma} \cong \mathcal{O}_{p}$.

Proof. We observe that the proof of proposition 5.7 shows that $\overline{\mathcal{C}}_{\infty, \mathfrak{f}_{\theta}}^{\chi} \cong \mathcal{O}_{p}[[\Gamma]]$ is a free $\mathcal{O}_{p}[[\Gamma]]$-module of rank 1 . This implies, as in [22, lemma 5.2.3], that $\left(\overline{\mathcal{C}}_{\infty, \mathfrak{f}_{\theta}}^{\chi} \otimes M_{\theta \mathbb{Z}_{p}}(w+l)\right)_{\Gamma} \cong \mathcal{O}_{p}$. The claim follows since the previous module is induced and hence the higher Tor-terms vanish. 
Corollary 5.11. The map

$$
\mathcal{R}_{\theta} \otimes \mathbb{Z}_{p} \rightarrow R \Gamma\left(\mathcal{O}_{S}, M_{\theta \mathbb{Z}_{p}}(w+l+1) \otimes \mathbb{Q}_{p}\right)[1]
$$

induced by $r_{p}$, gives an isomorphism

$$
\operatorname{det}_{\mathcal{O}_{p}} \mathcal{R}_{\theta} \cong \operatorname{det}_{\mathcal{O}_{p}} R \Gamma\left(\mathcal{O}_{S}, M_{\theta \mathbb{Z}_{p}}(w+l+1)\right)^{-1}
$$

Before stating the next theorem, let us recall all the hypothesis we used during the paper and that we will need:

$(\diamond \diamond \diamond)$ Let $p$ be a fix prime such that $p \nmid 6 N_{K / \mathbb{Q}} \mathfrak{f}$ (hence, in particular $p \nmid\left(\# \mathcal{O}_{K}^{*}\right)$, and $\left.p \nmid D_{K}\right)$. Consider $l$ a non-negative integer. Let $\left(a_{\theta}, b_{\theta}\right)$ be the infinite type of $\psi_{\theta}$, with $a_{\theta}, b_{\theta}$ non-negative integers with $w=a_{\theta}+b_{\theta} \geq 1$ such that $a_{\theta} \not \equiv b_{\theta} \bmod \left(\# \mathcal{O}_{K}^{*}\right)$ and $-w-2 l \leq-3$. Assume that $\mathcal{O}_{K}^{*} \rightarrow$ $\left(\mathcal{O}_{K} / \mathfrak{f}_{\theta}\right)^{*}$ is injective. Suppose moreover that the representation $\chi$ of $\Delta$ in $\operatorname{Hom}_{\mathcal{O}_{p}}\left(H_{e t}^{w}\left(M_{\theta} \times_{K} \bar{K}, \mathbb{Z}_{p}(w+l)\right), \mathcal{O}_{p}\right)$ is a good representation (see the definition in 4.3) which is not equal as $\Delta$-representation to the cyclotomic representation.

Theorem 5.12. Under the hypothesis $(\diamond \diamond \diamond)$ above, there is an $\mathcal{O}_{K}$-submodule $\mathcal{R}_{\theta} \subset H_{\mathcal{M}}^{w+1}\left(M_{\theta \mathbb{Q}}, \mathbb{Q}(w+l+1)\right)$ of rank 1 such that:

1. $\operatorname{det}_{\mathcal{O}_{K}\left[1 / D_{K}\right]}\left(r_{\mathcal{D}}\left(\mathcal{R}_{\theta} \otimes_{\mathcal{O}_{K}} \mathcal{O}_{K}\left[1 / D_{K}\right]\right)\right) \cong$

$$
L_{S}^{*}\left(\bar{\psi}_{\theta},-l\right) \operatorname{det}_{\mathcal{O}_{K}\left[1 / D_{K}\right]}\left(H_{B}^{w}\left(M_{\theta \mathbb{C}}, \mathbb{Z}(w+l)\right) \otimes_{\mathcal{O}_{K}} \mathcal{O}_{K}\left[1 / D_{K}\right]\right)
$$

in $\operatorname{det}_{\mathcal{O}_{K}\left[1 / D_{K}\right] \otimes \mathbb{R}}\left(H_{B}^{w}\left(M_{\theta \mathbb{C}}, \mathbb{Z}(w+l)\right) \otimes_{\mathcal{O}_{K}} \mathcal{O}_{K}\left[1 / D_{K}\right] \otimes \mathbb{R}\right)$.

2. The map $r_{p}$ induces an isomorphism

$$
\operatorname{det}_{\mathcal{O}_{K} \otimes \mathbb{Z}_{p}}\left(\mathcal{R}_{\theta}\right) \cong \operatorname{det}_{\mathcal{O}_{K} \otimes \mathbb{Z}_{p}}\left(R \Gamma\left(\mathcal{O}_{K}[1 / S], M_{\theta \mathbb{Z}_{p}}(w+l+1)\right)^{-1} .\right.
$$

Here

$$
L_{S}^{*}\left(\bar{\psi}_{\theta},-l\right)=\lim _{s \rightarrow-l} \frac{L_{S}\left(\bar{\psi}_{\theta}, s\right)}{s+l},
$$

and $S$ is the set of primes of $K$ dividing $p$ and the ones dividing $\mathfrak{f}_{\theta}$.

Moreover, if $r_{p}$ is injective on $\mathcal{R}_{\theta}$, the second part can be written as

$$
\begin{gathered}
\operatorname{det}_{\mathcal{O}_{K} \otimes \mathbb{Z}_{p}}\left(H^{1}\left(\mathcal{O}_{K}[1 / S], M_{\theta \mathbb{Z}_{p}}(w+l+1)\right) / r_{p}\left(\mathcal{R}_{\theta}\right)\right) \cong \\
\operatorname{det}_{\mathcal{O}_{K} \otimes \mathbb{Z}_{p}} H^{2}\left(\mathcal{O}_{K}[1 / S], M_{\theta \mathbb{Z}_{p}}(w+l+1)\right) .
\end{gathered}
$$

Proof. It is a direct consequence of the theorem 3.5 and the above corollary 5.11.

After taking the norm $N_{K / \mathbb{Q}}$, we obtain the following result.

Theorem 5.13. Under the assumption $(\diamond \diamond \diamond)$ above, there is a $\mathbb{Z}$-submodule $\mathcal{R}_{\theta}$ in $H_{\mathcal{M}}^{w+1}\left(M_{\theta \mathbb{Q}}, \mathbb{Q}(w+l+1)\right)$ of rank 2 such that:

1. The map $r_{\mathcal{D}} \otimes \mathbb{R}$ is an isomorphism restricted to $\mathcal{R}_{\theta} \otimes \mathbb{R}$.

2. $\operatorname{dim}_{\mathbb{Q}}\left(H_{B}^{w}\left(M_{\theta \mathbb{C}}, \mathbb{Q}(w+l)\right)\right)=\operatorname{ord}_{s=-l} L_{S}\left(M_{\theta \mathbb{Q}}, s\right)=2$. 
3. We have the equality

$$
\begin{gathered}
r_{\mathcal{D}}\left(\operatorname{det}_{\mathbb{Z}\left[1 / D_{K}\right]}\left(\mathcal{R}_{\theta} \otimes_{\mathcal{O}_{K}} \mathcal{O}_{K}\left[1 / D_{K}\right]\right)\right)= \\
L_{S}^{*}\left(M_{\theta \mathbb{Q}},-l\right) \operatorname{det}_{\mathbb{Z}\left[1 / D_{K}\right]}\left(H_{B}^{w}\left(M_{\theta \mathbb{C}}, \mathbb{Z}(w+l)\right) \otimes_{\mathcal{O}_{K}} \mathcal{O}_{K}\left[1 / D_{K}\right]\right)
\end{gathered}
$$

where

$$
L_{S}^{*}\left(M_{\theta \mathbb{Q}},-l\right)=\lim _{s \rightarrow-l} \frac{L_{S}\left(M_{\theta \mathbb{Q}}, s\right)}{(s+l)^{2}}
$$

and $S$ is the set of places of $K$ that divides $p$ and the places dividing the conductor $\mathfrak{f}_{\theta}$.

4. We have that

$$
\operatorname{det}_{\mathbb{Z}_{p}}\left(\mathcal{R}_{\theta} \otimes \mathbb{Z}_{p}\right)=\operatorname{det}_{\mathbb{Z}_{p}}\left(R \Gamma\left(\mathcal{O}_{K}[1 / S], M_{\theta \mathbb{Z}_{p}}(w+l+1)\right)\right)^{-1} .
$$

If $r_{p}$ is injective on $\mathcal{R}_{\theta}$, then $r_{p}\left(\operatorname{det}_{\mathbb{Z}_{p}}\left(\mathcal{R}_{\theta} \otimes \mathbb{Z}_{p}\right)\right)$ is a basis of the $\mathbb{Z}_{p}$-lattice

$$
\begin{gathered}
\operatorname{det}_{\mathbb{Z}_{p}}\left(R \Gamma\left(\mathcal{O}_{K}[1 / S], M_{\theta \mathbb{Z}_{p}}(w+l+1)\right)\right)^{-1} \\
\subset \operatorname{det}_{\mathbb{Q}_{p}}\left(R \Gamma\left(\mathcal{O}_{K}[1 / S], M_{\theta \mathbb{Z}_{p}}(w+l+1) \otimes \mathbb{Q}\right)[-1]\right) .
\end{gathered}
$$

Remark 5.14. Theorems 5.12 and 5.13 imply the weak p-part of the Tamagawa number conjecture for Hecke characters [19] for $K$ or $\mathbb{Q}$ coefficients respectively, up to the finiteness of $H_{p}^{2}:=H^{2}\left(\mathcal{O}[1 / S], M_{\theta \mathbb{Z}_{p}}(w+l+1)\right)$ and the bijectively of the Soule regulator map $r_{p}$. Concerning these requirements, we have the following.

1. If $p$ is a regular prime for the field $K(E[p])$, then $H_{p}^{2}$ is finite [3]. Moreover without any assumption, one obtains that for almost all $l$ this Galois cohomology group is finite [21, Theorem 12.4] or [4].

2. About the bijectively of the Soulé regulator map observe if $H_{p}^{2}$ is finite, similar arguments as in [22, §5.2.2] implies the injectivity for $(S o u l)_{p}$ and therefore $r_{p}$ is injective on $\mathcal{R}_{\theta} \otimes \mathbb{Q}_{p}$ by corollary [5.9. Therefore $r_{p}$ restricted to $\mathcal{R}_{\theta} \otimes \mathbb{Q}_{p}$ is an isomorphism [18, cor. 1].

Therefore for regular primes $p$, we obtain in full generality the weak p-part of the Tamagawa number conjecture for Hecke characters of imaginary quadratic fields.

\section{The remaining Tate twists}

\subsection{The remaining non-critical twists}

The value of the $L$-function at zero for $M_{\theta}(w+l+1)$ with $-w-2 l<-2$ is related with the first non-zero coefficient of the Taylor development at $-l$ of the $L$-function associated to $\bar{\psi}_{\theta}$ by the use of the functional equation of $L$-functions. The non-critical values associated to the Hecke character $\bar{\psi}_{\theta}$ (we restrict to the situation $\left.a_{\theta} \not \equiv b_{\theta}\left(\bmod \left|\mathcal{O}_{K}^{*}\right|\right)\right)$ are the integers $l$ such that $-l \leq \min \left(a_{\theta}, b_{\theta}\right)$ where $a_{\theta}, b_{\theta}$ are associated to the Hecke character $\psi_{\theta}$ (see [8, Theorem 1.4.1])

The general formulation of the Tamagawa number conjecture at the noncritical values following [19] assumes $w+l+1>w$ [19, Conjecture 2.2.7] because 
then one avoids the poles in the bad Euler factors, and therefore the assumption $l \geq 0$. But, for $M_{\theta}(w+l+1)$, there are no poles in the bad Euler factors, see Remark 2.5. Thus, we can study the Tamagawa number conjecture for $l<0$ using only the regulators maps.

In this section we construct elements in $K$-theory for $M_{\theta}(w+l+1)$ with $0<-l \leq \min \left(a_{\theta}, b_{\theta}\right)$ and we study the image of these elements by the Beilinson regulator map and the Soulé regulator map, obtaining the weak $p$-part of the Tamagawa number conjecture.

Deninger [8, pp.142-144] already constructed elements in $K$-theory for the motive $M_{\theta}(w+l+1)$ with $l<0$ non-critical and obtains their image by the Beilinson regulator map, proving the Beilinson conjecture. He constructed these elements in $K$-theory by use of a projector map $\underline{\mathcal{K}}_{\mathcal{M}}$ without using complex multiplication. The problem of his construction is that the Weil pairing appearing in $\S 5$ to a $E\left[p^{r}\right]$-torsion point $\widetilde{t_{r}}, \gamma\left(\widetilde{t_{r}}\right)=<\widetilde{t_{r}}, \widetilde{t_{r}}>$ is trivial and the arguments through $\S 5$ does not generalize in order to construct an Euler system to control the image by the Soule regulator map. We modify Deninger's projector map by $\mathcal{K}_{\mathcal{M}}^{\prime}$ (we use now complex multiplication), and we construct the elements in $K$-theory using $\mathcal{K}_{\mathcal{M}}^{\prime}$ and we reobtain Beilinson's conjecture. With this modification the arguments in the $p$-part of the weak Tamagawa number conjecture, i.e. the image by the Soulé regulator map of these $K$-theory elements $\S 4, \S 5$, apply straightforward obtaining the weak $p$-part of the Tamagawa number conjecture for $l<0$, Theorems 6.3 , 6.4.

\subsection{Modification of Deninger's projector map. Beilinson conjecture revisited.}

Let us fix $w \geq 1$ and $l<0$ such that $-w-2 l \leq-3$ with $0<-l \leq \min \left(a_{\theta}, b_{\theta}\right)$ and let us consider the motive $M_{\theta}(w+l+1)$. With the fixed embedding we have $\vartheta=\left(\lambda_{1}, \ldots, \lambda_{w}\right) \in \theta_{K}$ and set $I_{1}=\left\{i \mid \lambda_{i} \in \operatorname{Hom}_{K}(K, \mathbb{C})\right\}$ and $I_{2}=$ $\left\{i \mid \lambda_{i} \notin \operatorname{Hom}_{K}(K, \mathbb{C})\right\}$ and we have now that $0<|l| \leq \# I_{1}=a_{\theta}$ and $0<$ $|l| \leq \# I_{2}=b_{\theta}$, where $|l|$ is the absolute value. Denote by $\Delta=i d^{1} \times i d^{2}$ : $E \rightarrow E \times E$ the diagonal map and by $\Delta_{C M}=i d^{1, C M} \times i d^{2, C M}: E \rightarrow E \times E$ given by $e \mapsto\left(e,\left(\sqrt{d_{K}}\right) e\right)$ where we understand $\sqrt{d_{K}} \in \operatorname{End}(E)$. Let us choose exactly $\#|l|$ elements in the sets $I_{1}$ and $I_{2}$, denote their in increasing order $i_{1}, \ldots, i_{|l|} \in I_{1}$ and $j_{1}, \ldots, j_{|l|} \in I_{2}$. Let us define the projector map $p r$ : $E^{w+l} \rightarrow E^{w+2 l}$ by the projection of the first $w+2 l$-components of $E^{w+l}$ and define $\left(i d \times \Delta^{|l|}\right): E^{w+l} \rightarrow E^{w}$ (which it depends of the choice in the sets $I_{1}$ and $\left.I_{2}\right)$ by $\left(e_{1}, \ldots, e_{w+2 l}, e_{w+2 l+1}, \ldots, e_{w+l}\right) \mapsto\left(e_{\alpha_{1}}, \ldots, e_{\alpha_{w}}\right)$ where $e_{\alpha_{s}}$ is defined as follows:

- if $\alpha_{s}$ appears in one component of the set of tuples $L:=\left\{\left(i_{1}, j_{1}\right), \ldots,\left(i_{|l|}, j_{|l|}\right)\right\}$ then

$$
e_{\alpha_{s}}:=\left\{\begin{array}{l}
i d^{1}\left(e_{w+2 l+m}\right) \text { if } \alpha_{s}=i_{m} \\
i d^{2}\left(e_{w+2 l+m}\right) \text { if } \alpha_{s}=j_{m}
\end{array},\right.
$$

- in the other case, then it is defined by $e_{\alpha_{s}}:=e_{\tilde{n}}$ with $1 \leq \tilde{n} \leq w+2 l$ such that $\alpha_{s}=\tilde{n}+\sum 1$ where the sum runs the naturals that appear in some component of the elements of $L$ and which are lower than $\alpha_{s}$. $i d^{i, C M}$.

We define the map $\left(i d \times \Delta_{C M}^{|l|}\right)$ similar as $\left(i d \times \Delta^{|l|}\right)$ but replacing $i d^{i}$ by 
The projector map $\mathcal{K}_{\mathcal{M}}^{\prime}$ is defined by the commutative diagram

$$
\begin{aligned}
& H_{\mathcal{M}}^{2 l+w+1}\left(S y m^{2 l+w} h^{1} E, \mathbb{Q}(w+2 l+1)\right) \quad \stackrel{p r^{*}}{\longrightarrow} \quad H_{\mathcal{M}}^{2 l+w+1}\left(E^{2 l+w+|l|}, \mathbb{Q}(2 l+w+1)\right) \\
& \mathcal{K}_{\mathcal{M}}^{\prime} \downarrow \\
& H_{\mathcal{M}}^{w+1}\left(M_{\theta \mathbb{Q}}, \mathbb{Q}(w+l+1)\right) \quad \stackrel{e_{\theta}}{\longleftarrow} \quad H_{\mathcal{M}}^{w+1}\left(h^{1}(E)^{\otimes w}, \mathbb{Q}(l+w+1)\right) .
\end{aligned}
$$

Deninger defines a projector map $\underline{\mathcal{K}}_{\mathcal{M}}$ with a similar diagram as for our $\mathcal{K}_{\mathcal{M}}^{\prime}$ but replacing the map $\left(i d \times \Delta_{C M}^{|l|}\right)_{*}$ by the map $\left(i d \times \Delta^{|l|}\right)_{*}$.

Let us choose the element in $H_{\mathcal{M}}^{w+1}\left(M_{\theta \mathbb{Q}}, \mathbb{Q}(w+l+1)\right)$

$$
\Upsilon_{\theta}:=\mathcal{K}_{\mathcal{M}}^{\prime} \mathcal{E}_{\mathcal{M}}^{2 l+w}\left(N_{K(E[\mathfrak{f}]) / K}\left(\left(\Omega f^{-1}\right)\right)\right),
$$

where $\mathcal{E}_{\mathcal{M}}^{2 l+w}$ is the Eisenstein symbol, $f$ a generator of $\mathfrak{f}_{\theta}, \Omega$ the period of $E$ and $\left(\Omega f^{-1}\right)$ means the divisor in $\mathbb{Z}\left[E\left[\mathfrak{f}_{\theta}\right] \backslash 0\right]$.

The next result is a modification of Deninger's result [8, pp.143-145].

Theorem 6.1. Suppose $a_{\theta} \not \equiv b_{\theta} \bmod \left(\# \mathcal{O}_{K}^{*}\right)$ with $a_{\theta}, b_{\theta} \geq 0, l<0, w=a_{\theta}+b_{\theta}$, with $-w-2 l \leq-3$ and $-l \leq \min \left(a_{\theta}, b_{\theta}\right)$. Define, up to sign,

$$
\xi_{\theta, l}:=\frac{\left(\sqrt{d_{K}}\right)^{2 l}(2 l+w) ! L_{p}\left(\overline{\psi_{\theta}},-l\right)^{-1} \Phi\left(\mathfrak{f}_{\theta}\right)}{2^{-1} N_{K / \mathbb{Q}} \mathfrak{f}_{\theta}^{l} \psi_{\theta}\left(\rho_{\theta}\right) \Phi(\mathfrak{f})} \Upsilon_{\theta}
$$

which belongs to $H_{\mathcal{M}}^{w+1}\left(M_{\theta \mathbb{Q}}, \mathbb{Q}(w+l+1)\right)$ where $L_{p}\left(\overline{\psi_{\theta}},-l\right)$ means the product of the Euler factors of the primes above $p$ of $K$ at $-l$ (is well defined by Remark 2.5), and $\rho_{\theta}$ is the idèle of $K$ such that $v_{\mathfrak{q}}\left(\rho_{\mathfrak{q}}^{-1}-f^{-1}\right) \geq 0$ for $\mathfrak{q} \mid \mathfrak{f}_{\theta}$ and $v_{\mathfrak{q}}\left(\rho_{\mathfrak{q}}\right)=0$ in the other primes $\mathfrak{q}$. Then

$$
r_{\mathcal{D}}\left(\xi_{\theta, l}\right)=L_{S}^{*}\left(\overline{\psi_{\theta}},-l\right) \eta_{\theta},
$$

where $S$ are the set of primes of $K$ that divide $\mathfrak{f}_{\theta} p, \eta_{\theta}$ is an $\mathcal{O}_{K}\left[1 / D_{K}\right]$-basis for $H_{B}^{w}\left(M_{\theta \mathbb{C}}, \mathbb{Z}(w+l)\right) \otimes \mathcal{O}_{K} \mathcal{O}_{K}\left[1 / D_{K}\right]$ and $L_{S}^{*}\left(\bar{\psi}_{\theta},-l\right)=\lim _{s+l \rightarrow 0} \frac{L_{S}\left(\bar{\psi}_{\theta}, s\right)}{s+l}$.

Proof. We will follow closely Deninger's papers [7] and [8], we follow also in this proof his notation where his $n$ is our $w+2 l$. Deninger defines the element $\xi_{\theta, l}$ from $\underline{\mathcal{K}}_{\mathcal{M}} \mathcal{E}_{\mathcal{M}}^{2 l+w}\left(N_{K(E[\mathfrak{f}]) / K}\left(\left(\Omega f^{-1}\right)\right)\right)$ instead of $\Upsilon_{\theta}$. We modify only the calculation in [8, (2.13)Lemma] for $\mathcal{K}_{\mathcal{M}}^{\prime}$ instead of $\underline{\mathcal{K}}_{\mathcal{M}}$. One obtains (up to sign)

$$
\begin{gathered}
\frac{1}{(2 \pi i)^{w}} \int_{E^{w}} \tilde{\mathcal{K}}_{\mathcal{D}}^{\prime}(\tilde{\xi}) \wedge d z^{(\underline{\varepsilon})}= \\
B_{\underline{\varepsilon} \sqrt{d_{K}}|l|}\left(\begin{array}{c}
n \\
n+|l|-|\underline{\varepsilon}|
\end{array}\right)^{-1} A(\Gamma)^{n+|l|} c_{n+|l|-|\underline{\varepsilon}|}
\end{gathered}
$$

see the calculation at the top of [7, p.63]. To precise the sgn we should control the chosen order of the factors of the map $\left(i d \times \Delta_{C M}^{|l|}\right)$, but for our interest is unnecessary. Then the argument [8, p.143-144] applies in our situation obtaining,

$$
r_{\mathcal{D}}\left(\Upsilon_{\theta}\right)=t_{\theta} L^{*}\left(\bar{\psi}_{\theta},-l\right) \eta_{\theta}
$$

where $t_{\theta}$ is given by $\frac{2^{-1} N_{K / \mathbb{Q}} \mathfrak{f}_{\theta}^{l} \psi_{\theta}\left(\rho_{\theta}\right) \Phi(\mathfrak{f})}{\left(\sqrt{d_{K}}\right)^{2 l}(2 l+w) ! \Phi\left(\mathfrak{f}_{\theta}\right)}$ (up to sign). By Remark 2.5 we can introduce the Euler factors above $p$ in the constant factor $t_{\theta}$, obtaining the statement. 


\subsection{The weak Tamagawa number conjecture for $l<0$}

Following $\S 3$ we define for $l<0$ the constructible module by

$$
\mathcal{R}_{\theta}:=\xi_{\theta, l} \mathcal{O}_{K}
$$

where $\xi_{\theta, l}$ is defined in theorem 6.1. Let us observe that with this notation we can follow straightforward all the results and proofs of $\S 3$ and $\S 4$. In $\S 5$ we need to compute $\mathcal{K}_{\mathcal{M}}^{\prime} \circ \mathcal{E}_{\mathcal{M}}^{w+2 l}\left(N_{K(E[\mathfrak{f}]) / K}\left(\Omega f^{-1}\right)\right)$. We remember that we suppose once and for all that $p \nmid D_{K}$. Denote by $e=\left(\widetilde{t_{r}}\right)_{r}$ and element of the Tate module $T_{p} E$ where $\widetilde{t_{r}} \in E\left[p^{r}\right]$ a $p^{r}$-torsion point for $E$.

Lemma 6.2. The realization on Galois cohomology of the projector map $\mathcal{K}_{\mathcal{M}}^{\prime}$ has the property, $\mathcal{K}_{\mathcal{M}}^{\prime}\left({\widetilde{t_{r}}}^{\otimes 2 l+w}\right)=e_{\theta}\left(\otimes^{w} \widetilde{t_{r}}\right) \otimes \gamma\left(\widetilde{t_{r}}\right)^{l}$ where $\gamma\left(\widetilde{t_{r}}\right)=<\widetilde{t_{r}}, \sqrt{d_{K}} \widetilde{t_{r}}>$.

Proof. Observe first that the projector map $\mathcal{K}_{\mathcal{M}}^{\prime}$ is $e_{\theta} \circ\left(i d \times \Delta_{C M}^{|l|}\right)_{*} \circ p r^{*}$. Let us take $\delta_{*}:=\left(i d \times \Delta_{C M}^{|l|}\right)_{*} \circ p r^{*}$ and observe that its transpose $\delta^{*}=p r_{*} \circ\left(i d \times \Delta_{C M}^{|l|}\right)^{*}$ is part of the definition of $\mathcal{K}_{\mathcal{M}}=e_{\theta} \circ \delta^{*}$ with $\underline{l}:=|l|>0$ given at [22]. We want only to study these projector maps on the Galois cohomology. Denote by $\mathcal{H}_{\mathbb{Q}_{p}}$ the étale realization of $h^{1}(E)(1)$ and observe that there is an isomorphism $\mathcal{H}_{\mathbb{Q}_{p}}^{*}(1) \cong \mathcal{H}_{\mathbb{Q}_{p}}$, since $\left(h^{1}(E)(1)\right)^{*}=h_{1}(E)(-1) \cong h^{1}(E)(1)(-1)=h^{1}(E)$. The map $\delta^{*}$ is given by

$$
H^{1}\left(\mathcal{O}_{S}, \operatorname{Sym}^{2 \underline{l}+\underline{w}}\left(\mathcal{H}_{\mathbb{Q}_{p}}\right)(1)\right) \rightarrow H^{1}\left(\mathcal{O}_{S}, \operatorname{Sym} \underline{\underline{w}}\left(\mathcal{H}_{\mathbb{Q}_{p}}\right)(\underline{l}+1)\right),
$$

and because the map $\delta_{*}$ is the transpose for the map $\delta^{*}$, up to Tate twist by $\underline{w}+\underline{l}$, it is represented by global Tate duality by,

$$
H^{1}\left(\mathcal{O}_{S}, \operatorname{Sym} \underline{\underline{w}}\left(\mathcal{H}_{\mathbb{Q}_{p}}\right)^{*}(-\underline{l}-1)(1)\right) \rightarrow H^{1}\left(\mathcal{O}_{S}, \operatorname{Sym}^{2 \underline{l}+\underline{w}}\left(\mathcal{H}_{\mathbb{Q}_{p}}\right)^{*}(-1)(1)\right) .
$$

Is known 22 that

$$
\delta^{*}\left(\lim _{r}\left(\otimes \underline{2} \underline{\underline{l}}+\underline{w} \widetilde{t_{r}}\right)\right)=\lim _{\leftarrow_{r}}\left(\left(\otimes \underline{w} \widetilde{t_{r}}\right) \gamma\left(\widetilde{t}_{r}\right)^{\underline{l}}\right)
$$

write this equality also by $\delta^{*}(\otimes \underline{w}+2 \underline{l} v)=(\otimes \underline{w} v) \gamma(v) \underline{\underline{l}}$. Take now the dual map by $\operatorname{Hom}\left(, \mathbb{Z}_{p}\right)$ and with the identification $T_{p} E \cong H o m\left(T_{p} E, \mathbb{Z}_{p}(1)\right)$, we obtain

$$
(\otimes \underline{w} v(-1)) \gamma(v)^{-\underline{l}} \mapsto \otimes \underline{w}+2 \underline{l} v(-1)
$$

twisting now by $\underline{w}+\underline{l}$ we arrive to the definition for $\delta_{*}$ and,

$$
\delta_{*}(\otimes \underline{w} v) \mapsto(\otimes \underline{w}+2 \underline{l} v) \gamma(v)^{-\underline{l}} .
$$

Now take this equality at level $r, w=\underline{w}+2 \underline{l}, l=-\underline{l}$, and apply the idempotent $e_{\theta}$ to finish.

After the lemma 6.2 all the results of $\S 5$ and the proofs of $\S 5$ follow straightforward up to a power of 2 and $D_{K}$, (the reader could make these modifications which follow only from our definition of $\mathcal{R}_{\theta}$ ). Therefore we obtain the weak $p$ part of the Tamagawa number conjecture with $K$-coefficients and $\mathbb{Q}$-coefficients, under standard hypothesis from Iwasawa theory for imaginary quadratic fields:

$(* * *)$ Let $p$ be a fix prime such that $p \nmid 6 N_{K / \mathbb{Q}} \mathfrak{f}$. Suppose that $\psi_{\theta}$ has infinity type $\left(a_{\theta}, b_{\theta}\right)$ with $a_{\theta}, b_{\theta}$ non-negative integers, such that $a_{\theta} \not \equiv b_{\theta} \bmod \left(\# \mathcal{O}_{K}^{*}\right)$ 
and $w=a_{\theta}+b_{\theta} \geq 1$ verifies $-w-2 l \leq-3$ with $l<0$ and $-l \leq \min \left(a_{\theta}, b_{\theta}\right)$. Suppose that $\mathcal{O}_{K}^{*} \rightarrow\left(\mathcal{O}_{K} / \mathfrak{f}_{\theta}\right)^{*}$ is injective. Suppose moreover that the representation $\chi$ of $\operatorname{Gal}(K(E[p]) / K)$ in $\operatorname{Hom}_{\mathcal{O}_{p}}\left(H^{w}\left(M_{\theta} \times_{K} \bar{K}, \mathbb{Z}_{p}(w+l)\right), \mathcal{O}_{p}\right)$ is a good representation which is not equal as $\Delta$-representation to the cyclotomic representation.

Theorem 6.3. Assume hypotheses (***). Then, there is an $\mathcal{O}_{K}$-submodule $\mathcal{R}_{\theta} \subset H_{\mathcal{M}}$ of rank 1 such that:

1. $\operatorname{det}_{\mathcal{O}_{K}\left[1 / D_{K}\right]}\left(r_{\mathcal{D}}\left(\mathcal{R}_{\theta} \otimes_{\mathcal{O}_{K}} \mathcal{O}_{K}\left[1 / D_{K}\right]\right)\right) \cong$

$$
L_{S}^{*}\left(\bar{\psi}_{\theta},-l\right) \operatorname{det}_{\mathcal{O}_{K}\left[1 / D_{K}\right]}\left(H_{B}^{w}\left(M_{\theta \mathbb{C}}, \mathbb{Z}(w+l)\right) \otimes_{\mathcal{O}_{K}} \mathcal{O}_{K}\left[1 / D_{K}\right]\right)
$$

in $\operatorname{det}_{\mathcal{O}_{K}\left[1 / D_{K}\right] \otimes \mathbb{R}}\left(H_{B}^{w}\left(M_{\theta \mathbb{C}}, \mathbb{Z}(w+l)\right) \otimes_{\mathcal{O}_{K}} \mathcal{O}_{K}\left[1 / D_{K}\right] \otimes \mathbb{R}\right)$.

2. The map $r_{p}$ induces an isomorphism

$$
\operatorname{det}_{\mathcal{O}_{K} \otimes \mathbb{Z}_{p}}\left(\mathcal{R}_{\theta}\right) \cong \operatorname{det}_{\mathcal{O}_{K} \otimes \mathbb{Z}_{p}}\left(R \Gamma\left(\mathcal{O}_{K}[1 / S], M_{\theta \mathbb{Z}_{p}}(w+l+1)\right)^{-1} .\right.
$$

Here

$$
L_{S}^{*}\left(\bar{\psi}_{\theta},-l\right)=\lim _{s \rightarrow-l} \frac{L_{S}\left(\bar{\psi}_{\theta}, s\right)}{s+l},
$$

and $S$ is the set of primes of $K$ dividing $p$ and the ones dividing $\mathfrak{f}_{\theta}$.

Moreover, if $r_{p}$ is injective on $\mathcal{R}_{\theta}$, the second part can be written as

$$
\begin{gathered}
\operatorname{det}_{\mathcal{O}_{K} \otimes \mathbb{Z}_{p}}\left(H^{1}\left(\mathcal{O}_{K}[1 / S], M_{\theta \mathbb{Z}_{p}}(w+l+1)\right) / r_{p}\left(\mathcal{R}_{\theta}\right)\right) \cong \\
\operatorname{det}_{\mathcal{O}_{K} \otimes \mathbb{Z}_{p}} H^{2}\left(\mathcal{O}_{K}[1 / S], M_{\theta \mathbb{Z}_{p}}(w+l+1)\right) .
\end{gathered}
$$

Theorem 6.4. Suppose hypotheses $(* * *)$.

Then, there is a $\mathbb{Z}$-submodule $\mathcal{R}_{\theta}$ in $H_{\mathcal{M}}$ of rank 2 such that:

1. The map $r_{\mathcal{D}} \otimes \mathbb{R}$ is an isomorphism restricted to $\mathcal{R}_{\theta} \otimes \mathbb{R}$.

2. $\operatorname{dim}_{\mathbb{Q}}\left(H_{B}^{w}\left(M_{\theta \mathbb{C}}, \mathbb{Q}(w+l)\right)\right)=\operatorname{ord}_{s=-l} L_{S}\left(M_{\theta \mathbb{Q}}, s\right)=2$.

3. We have the equality

$$
\begin{gathered}
r_{\mathcal{D}}\left(\operatorname{det}_{\mathbb{Z}\left[1 / D_{K}\right]}\left(\mathcal{R}_{\theta} \otimes_{\mathcal{O}_{K}} \mathcal{O}_{K}\left[1 / D_{K}\right]\right)\right)= \\
L_{S}^{*}\left(M_{\theta \mathbb{Q}},-l\right) \operatorname{det}_{\mathbb{Z}\left[1 / D_{K}\right]}\left(H_{B}^{w}\left(M_{\theta}, \mathbb{Z}(w+l)\right) \otimes_{\mathcal{O}_{K}} \mathcal{O}_{K}\left[1 / D_{K}\right]\right)
\end{gathered}
$$

where

$$
L_{S}^{*}\left(M_{\theta \mathbb{Q}},-l\right)=\lim _{s \rightarrow-l} \frac{L_{S}\left(M_{\theta \mathbb{Q}}, s\right)}{(s+l)^{2}}
$$

and $S$ is the set of places of $K$ that divides $p$ and the places dividing the conductor $\mathfrak{f}_{\theta}$.

4. We have that

$$
\operatorname{det}_{\mathbb{Z}_{p}}\left(\mathcal{R}_{\theta} \otimes \mathbb{Z}_{p}\right)=\operatorname{det}_{\mathbb{Z}_{p}}\left(R \Gamma\left(\mathcal{O}_{K}[1 / S], M_{\theta \mathbb{Z}_{p}}(w+l+1)\right)\right)^{-1} .
$$

If $r_{p}$ is injective on $\mathcal{R}_{\theta}$, then $r_{p}\left(\operatorname{det}_{\mathbb{Z}_{p}}\left(\mathcal{R}_{\theta} \otimes \mathbb{Z}_{p}\right)\right)$ is a basis of the $\mathbb{Z}_{p}$-lattice

$$
\begin{gathered}
\operatorname{det}_{\mathbb{Z}_{p}}\left(R \Gamma\left(\mathcal{O}_{K}[1 / S], M_{\theta \mathbb{Z}_{p}}(w+l+1)\right)\right)^{-1} \\
\subset \operatorname{det}_{\mathbb{Q}_{p}}\left(R \Gamma\left(\mathcal{O}_{K}[1 / S], M_{\theta \mathbb{Z}_{p}}(w+l+1) \otimes \mathbb{Q}\right)[-1]\right) .
\end{gathered}
$$




\section{$7 \quad$ Some explicit examples}

Observe first if we consider the Hecke character associated to the idempotent $e_{\theta}$ with infinite type $(1,0)$, then, Theorem 5.12 is exactly [22, Theorem 1.1.5] (hypothesis $(\diamond \diamond \diamond)$ for the infinite type $(1,0)$ coincides with the ones that appears in [22, Theorem 1.1.5], use [22, Corollary 2.2.11], Lemmata 4.5, 4.6, 5.5] and comments after Definition 4.3).

Let us give examples of Hecke characters of infinite type different to $(1,0)$. In the following, take $E$ the elliptic curve $y^{2}=4 x^{3}-4 x$ defined over $K=\mathbb{Q}(i)$ and the $e_{\theta}$ 's are defined from this fixed $E$. $E$ is an elliptic curve with CM by $\mathbb{Z}[i]$, and for $p \geq 5$ we have $p \nmid 6 N_{K / \mathbb{Q}} \mathfrak{f}$.

Let us consider $e_{\theta}$ with infinite type $(a, b)$ satisfying $a \not \equiv b(\bmod 2), a>b \geq 0$ and $-(a+b)-2 l \leq-3$ with $-l \leq b$. Take $p$ such that it splits in $\mathbb{Q}(i)$ and $p-1>\max (3, a-b)$. We have that $e_{\theta}$ and $p$ satisfy all the hypothesis $(\diamond \diamond \diamond)$ and $(* * *)$, with the exception of the condition (A) in Definition 4.3, (use Lemma 4.6 and with a similar proof done for Lemma 5.5 one obtains that $(\mathbb{Z}[i])^{*} \rightarrow\left(\mathbb{Z}[i] / \mathfrak{f}_{\theta}\right)^{*}$ is injective if $\left.\left(\# \mathcal{O}_{K}^{*}, a-b\right)=1\right)$. We impose $(a-b, p-1)=1$ to ensure that $e_{\theta}$ and $p$ satisfy the condition (A) (see Lemma 4.5). For such $e_{\theta}$ and $p$ we obtain the conclusion of theorems 5.12, 5.13, 6.3 and 6.4.

For an explicit example take $e_{\theta_{1}}$ with infinite type $(3,0)$ and $p=5$, then all hypothesis $(\diamond \diamond \diamond)$ are satisfied and moreover $p=5$ is a regular prime for $\mathbb{Q}(i)$ (see [28, p.33]) therefore by theorem 5.13 (and remark 5.14) we get the weak 5-part of the Tamagawa number conjecture for the dual of the motive $M_{\theta_{1}}(3+l+1)$ twisted by 1 (the special value for the motive $M$ is the special value $L(M, 0)$, and in our formulation we get the special value $\left.L\left(\bar{\psi}_{\theta},-l\right)=L\left(\left(M_{\theta}(w+l+1)\right)^{2}(1), 0\right)\right)$. By use of the functional equation between the motive and its dual twisted by 1 and good compatibilities, one should obtain the 5-part of the Tamagawa number conjecture for the motive $M_{\theta_{1}}(3+l+1)$ for any $l \geq 0$.

For another explicit example, take $e_{\theta_{2}}$ with infinity type $(a, b)=(3,2)$ and $p=5$, and we get the weak 5-part of the Tamagawa number conjecture for $\left(M_{\theta_{2}}(3+2+l+1)\right)^{c}(1)$ for $l \geq-1$ by theorems 5.13 and 6.4 .

\section{Acknowledgement}

I am indebt with X.Xarles. I would like to thank him for his useful comments and suggestions and moreover to give me energy to try to make me enjoy another time doing mathematical research. It is also a big pleasure to thank G. Kings for many discussions, C. Deninger for introducing me in the $L$-function world and K. Rubin for clarifying a doubt on elliptic units. Finally I thank the referees for their comments and suggestions.

\section{References}

[1] F. Bars : On the Tamagawa number conjecture for elliptic curves with CM over $\mathbb{Q}$. J. Number Theory 95, no. 2, 190-208 (2002).

[2] F. Bars: On the Tamagawa number conjecture. Thesis Universitat Autònoma de Barcelona, may 2001. http://www.tdcat.cesca.es/handle/10803/3072 
[3] F. Bars: On Jannsen's conjecture for Hecke characters of imaginary quadratic fields. Publ. Mat. vol. extra (2007), 29-42, Proceedings of the Primera Jornadas de Teoría de Números.

[4] F. Bars: The weak Leopoldt's conjecture for Hecke characters of imaginary quadratic fields. Journal of Algebra 319, 1954-1970 (2008).

[5] S. Bloch and K. Kato: L-functions and Tamagawa numbers of motives. In: P. Cartier et al. eds.: Grothendieck Festschrift Vol. I., 333-400, Birkhäuser (1990).

[6] D. Burns and C. Greither: On the equivariant Tamagawa number conjecture for Tate motives. Invent. Math. 153, 303-359 (2003).

[7] C. Deninger: Higher regulators and Hecke $L$-series of imaginary quadratic fields I. Invent. Math. 96, 1-69 (1989).

[8] C. Deninger: Higher regulators and Hecke $L$-series of imaginary quadratic field II. Ann. of Math.(2) 132, 131-155 (1990).

[9] E. de Shalit: Iwasawa Theory of Elliptic Curves with Complex Multiplication. Persp. in Mathematics 3, Acad.Press (1987).

[10] J.-M. Fontaine: Valeurs spéciales des fonctions $L$ des motifs. Séminaire Bourbaki, vol. 1991/92. Astérisque 206, exp. 751,4,205-249 (1992).

[11] J.-M. Fontaine and B. Perrin-Riou: Autour des conjectures de Bloch et Kato: cohomologie galoisienne et valeurs de fonctions $L$. In: Motives (Seetle) Proc. Symp. Pure Math. 55, 599-706 (1994).

[12] T. Geisser: A p-adic analogue of Beilinson's conjectures for Hecke characters of imaginary quadratic fields. Schriftenreihe des Math.Inst. der Universität Münster, 3 Serie, Helft 14(1995) Thesis.

[13] T. Geisser: $p$-adic $K$-theory of Hecke characters of imaginary quadratic fields and an analogue of Beilinson's conjectures. Duke Math. J. 86, no.2, 197-338 (1997).

[14] L. Guo: On the Bloch-Kato conjecture for Hecke $L$-functions. Journal of Number Theory 57, 340-365 (1996).

[15] B. Han: On Bloch-Kato conjecture of Tamagawa numbers for Hecke characters of imaginary quadratic number field. PhD Chicago University, 1997.

[16] M. Harrison: On the conjecture of Bloch-Kato for Grössencharacters over $\mathbb{Q}(i)$. PhD Thesis, Cambridge University 1993.

[17] A. Huber, G. Kings: Bloch-Kato conjecture and Main conjecture of Iwasawa Theory for Dirichlet characters. Duke Math. J. 119, no. 3, 393-464 (2003).

[18] U. Jannsen: On the $l$-adic cohomology of varieties over number fields and its Galois cohomology. In: Ihara et al. (eds.): Galois groups over $\mathbb{Q}$, MSRI Publication 16, 315-360 (1989). 
[19] K. Kato: Lectures on the approach to Iwasawa theory for Hasse-Weil Lfunctions via $B_{d R}$. In Arithmetic Algebraic Geometry (Trento, 1991), LNM 1553, 50-163 (1993).

[20] K. Kato: Iwasawa theory and p-adic Hodge theory. Kodai Math. J. 16, 1-31 (1993).

[21] K. Kato: p-adic Hodge theory and values of zeta functions of modular forms. Cohomologies $p$-adiques et applications arithmétiques, III. Astérisque 295, ix , 117-290, (2004).

[22] G. Kings: The Tamagawa number conjecture for CM elliptic curves. Invent. math. 143, 571-627 (2001).

[23] K. Kimura: Special values of Hecke $L$-functions of imaginary quadratic fields and explicit reciprocity law. Master's thesis, University of Tokyo, (1993).

[24] K. Rubin: The "main conjectures" of Iwasawa theory for imaginary quadratic fields. Invent. math. 103, 25-68 (1991).

[25] K. Rubin: Elliptic curves with complex multiplication and the conjecture of Birch and Swinnerton-Dyer, in: Arithmetic Theory of Elliptic Curves, LNM 1716, 167-234 (1999).

[26] C.Soulé: p-adic K-theory of elliptic curves. Duke Math. J. 54, 249-269 (1987).

[27] T. Tsuji: Explicit reciprocity law and formal moduli for Lubin-Tate formal groups. J.reine angew. Math. 569, 103-173 (2004).

[28] Yager, R.I.: A Kummer criterion for imaginary quadratic fields. Compositio Math., vol. 47, fasc. 1, 31-42 (1982).

Francesc Bars Cortina

Depart. Matemàtiques, Edifici C,

Universitat Autònoma de Barcelona.

08193 Bellaterra.

Catalonia. Spain

E-mail: francesc@mat.uab.cat, 